\title{
Docosahexaenoic Acid Induces Cell Death in Human Non-Small Cell Lung Cancer Cells by Repressing mTOR via AMPK Activation and PI3K/Akt Inhibition
}

\author{
Nayeong Kim,,2 Soyeon Jeong, ${ }^{1,2}$ Kaipeng Jing, ${ }^{3}$ Soyeon Shin, ${ }^{1,2}$ \\ Soyeon Kim, ${ }^{1,2}$ Jun-Young Heo, ${ }^{1}$ Gi-Ryang Kweon, ${ }^{1}$ Seung-Kiel Park, \\ Tong Wu, ${ }^{4}$ Jong-Il Park, ${ }^{1}$ and Kyu Lim ${ }^{1,2,5}$ \\ ${ }^{1}$ Department of Biochemistry, School of Medicine, Chungnam National University, Daejeon 301-747, Republic of Korea \\ ${ }^{2}$ Infection Signaling Network Research Center, School of Medicine, Chungnam National University, Daejeon 301-747, Republic of Korea \\ ${ }^{3}$ Stem Cell Research and Cellular Therapy Center, Affiliated Hospital of Guangdong Medical College, Zhanjiang 524001, China \\ ${ }^{4}$ Department of Pathology and Laboratory Medicine, Tulane University School of Medicine, New Orleans, LA 70112, USA \\ ${ }^{5}$ Cancer Research Institute, School of Medicine, Chungnam National University, Daejeon 301-747, Republic of Korea
}

Correspondence should be addressed to Kyu Lim; kyulim@cnu.ac.kr

Received 23 September 2014; Revised 22 December 2014; Accepted 22 December 2014

Academic Editor: Sung-Hoon Kim

Copyright (C) 2015 Nayeong Kim et al. This is an open access article distributed under the Creative Commons Attribution License, which permits unrestricted use, distribution, and reproduction in any medium, provided the original work is properly cited.

\begin{abstract}
The anticancer properties and mechanism of action of omega-3 polyunsaturated fatty acids ( $\omega 3$-PUFAs) have been demonstrated in several cancers; however, the mechanism in lung cancer remains unclear. Here, we show that docosahexaenoic acid (DHA), a $\omega 3$ PUFA, induced apoptosis and autophagy in non-small cell lung cancer (NSCLC) cells. DHA-induced cell death was accompanied by AMP-activated protein kinase (AMPK) activation and inactivated phosphatidylinositol 3-kinase (PI3K)/Akt/mammalian target of rapamycin (mTOR) signaling. Knocking down AMPK and overexpressing Akt increased mTOR activity and attenuated DHAinduced cell death, suggesting that DHA induces cell death via AMPK- and Akt-regulated mTOR inactivation. This was confirmed in Fat-1 transgenic mice, which produce $\omega 3$-PUFAs. Lewis lung cancer (LLC) tumor cells implanted into Fat-1 mice showed slower growth, lower phospho-Akt levels, and higher levels of apoptosis and autophagy than cells implanted into wild-type mice. Taken together, these data suggest that DHA-induced apoptosis and autophagy in NSCLC cells are associated with AMPK activation and PI3K/Akt inhibition, which in turn lead to suppression of mTOR; thus $\omega 3$-PUFAs may be utilized as potential therapeutic agents for NSCLC treatment.
\end{abstract}

\section{Introduction}

Lung cancer is the main cause of cancer-related death worldwide. According to the latest statistics from the United States National Cancer Institute, it is estimated that 224,210 Americans will be diagnosed with lung cancer in 2014 [1]. There are two types of lung cancer, namely, small-cell lung cancer (SCLC) and non-small-cell lung cancer (NSCLC), with more than $80 \%$ of lung cancer cases being NSCLC [2]. Because NSCLC is much less sensitive to chemotherapy than SCLC [3], a new approach for treating NSCLC is required.

Autophagy is a lysosome-associated degradation process that is characterized by the formation of double-membraned autophagosomes, which encapsulate cytoplasmic constituents [4-6]. The degraded components can then be used for energy production and other cellular processes [7]. Autophagy-related (Atg) genes, such as Atg12 and Atg5, are key molecules in regulation of autophagy. Atg12 constitutively associates with Atg5 to form Atg12-Atg5 conjugate, which is essential for the formation of lapidated microtubule-associated light chain 3 (LC3-II, a mammalian homolog of Atg8II) and autophagosomes $[8,9]$. The most potent inhibitor of autophagy is mammalian target of rapamycin (mTOR), which acts upstream of Atg proteins to regulate cell growth/ proliferation, survival, protein and lipid synthesis, lysosome biogenesis, and cytoskeletal organization [10-12]. mTOR is 
activated by Akt, a downstream product of phosphatidylinositol 3-kinase (PI3K), whereas the major negative regulator of mTOR is $5^{\prime}$ AMP-activated protein kinase (AMPK), which regulates intracellular energy status by sensing the AMP/ATP ratio [13-15]. mTOR utilizes p70 ribosomal S6 kinases 1 (S6K1) and eIF4E-binding protein 1 (4E-BP1) as substrates [16]. Inhibiting mTOR not only blocks the phosphorylation of S6K1 and 4E-BP1, but also induces autophagy [16-18].

Docosahexaenoic acid (DHA), an omega-3 polyunsaturated fatty acid ( $\omega 3$-PUFA), induces apoptosis in tumor cells by regulating several key signaling mediators, including $\mathrm{Wnt} / \beta$-catenin [19], p53-regulated activator protein 1 [20], and mitogen-activated protein kinase $[21,22]$. In addition, we have previously reported that DHA simultaneously induces apoptosis and autophagy in human cervical cancer as well as prostate cancer cells, and this process involves mTOR repression $[23,24]$. Despite several studies describing that the anti-lung cancer activity of DHA may be dependent on its proapoptosis effect [21, 25-27], it is still unclear whether it also induces autophagy in vitro and in vivo.

Here, we examined the mechanism(s) underlying DHAinduced cell death in human NSCLC cells. The results showed that DHA reduced cell viability and induced both apoptosis and autophagy. Moreover, DHA-induced cell death was associated with AKT-mTOR signaling inhibition and AMPK activation. Similarly, lung cancer cells implanted into Fat-1 transgenic mice exhibited higher levels of apoptosis, a higher autophagy index, and lower levels of phospho-AKT than cells implanted into wild-type mice. Taken together, these data show that DHA induces apoptosis and autophagy through AKT-mTOR signaling inhibition and AMPK activation, suggesting that $\omega 3$-PUFAs may be a potential treatment for human NSCLC.

\section{Materials and Methods}

2.1. Chemicals and Antibodies. DHA (Cayman Chemical, Ann Arbor, MI, USA; dissolved in absolute ethanol), chloroquine (CQ, Sigma, ST Louis, MO, USA), Bafilomycin A1 (Tocris, Bristol, UK; dissolved in phosphate buffered saline (Sigma)), and rapamycin (Tocris; dissolved in dimethyl sulfoxide (DMSO; Sigma)) were stored at $-20^{\circ} \mathrm{C}$ before use.

The following antibodies were used in this study: PI3K (p85), Akt, phospho-Akt (Ser473), phosphophosphatase and tensin homolog deleted on chromosome 10 (PTEN), AMPK, phospho-AMPK (Thr172), phospho-mTOR (Ser2448), phospho-S6K1 (Thr389), Atg5, Atg7, 4E-BP1, and LC3B (all Cell Signaling Technology, Beverly, MA, USA) and poly(ADP-ribose) polymerase- (PARP-) 1/2 (H-250), phospho-Akt (Thr308), p27 (C-19), and actin (I-19)-R (all Santa Cruz, CA, USA). Goat anti-rabbit and goat anti-mouse secondary antibodies were purchased from Calbiochem (Billerica, MA, USA).

2.2. Cells Lines and Cultures. Human NSCLC A549 cells and H1299 cells were purchased from American Type Cell Culture Collection (Rockville, MD, USA) and maintained in Dulbecco's Modified Eagle's Medium (DMEM; GIBCO,
Grand Island, NY, USA) supplemented with 10\% heatinactivated fetal bovine serum (FBS; GIBCO), penicillin, and streptomycin (GIBCO) in a humidified $5 \% \mathrm{CO}_{2}$ atmosphere at $37^{\circ} \mathrm{C}$.

2.3. Cell Viability Assay. Cell viability was determined using thiazolyl blue tetrazolium bromide (MTT; Sigma). Cells were seeded into 96 -well plates $\left(7 \times 10^{3}\right.$ per well) and incubated for $18 \mathrm{~h}$ at $37^{\circ} \mathrm{C}$ to allow adherence. The cells were then incubated with serum-free medium for $24 \mathrm{~h}$ and then treated with DHA for another $24 \mathrm{~h}$. The cells were then incubated with MTT for $2 \mathrm{~h}$ and the formazan products dissolved in DMSO. Absorbance was assayed at $570 \mathrm{~nm}$ in a spectrophotometer (Thermo Fisher Scientific, Waltham, MA, USA).

2.4. Western Blot Analysis. Western blot analysis was performed as described previously [28]. Briefly, cell lysates $(30 \mu \mathrm{g})$ were resolved by $6-15 \%$ sodium dodecyl sulfatepolyacrylamide gels and then transferred to polyvinylidene difluoride membranes (Millipore, Billerica, MA, USA). The membranes were then blocked in 5\% (w/v) skimmed-milk for $1 \mathrm{~h}$, followed by incubation with appropriate primary antibodies (diluted 1:1000-1:5000) overnight at $4^{\circ} \mathrm{C}$. Bound antibodies were detected with peroxidase-conjugated goat anti-rabbit or goat anti-mouse secondary antibodies and the blots developed using enhanced chemiluminescence (Millipore).

2.5. Apoptosis Analysis. Apoptosis was measured using a terminal deoxynucleotidyl transferase dUTP nick end labeling (TUNEL) assay, flow cytometry, and an Annexin V assay as described previously [23, 29].

2.6. Immunofluorescence Analysis. Cells were grown to $70 \%$ confluence in growth medium for $18 \mathrm{~h}$ before infection with recombinant adenoviruses expressing GFP-tagged LC3 (GFP-LC3, a gift from Professor Chang Deok Kim, Chungnam National University, Korea). DHA was then added to the infected cell cultures for $24 \mathrm{~h}$. The cells were observed by an Olympus iX70 fluorescence microscope.

2.7. Small Interfering RNAs (siRNAs) and Transfection. siRNAs targeting Atg5 and Atg7 were purchased from Invitrogen (Camarillo, CA, USA). A nontargeting control siRNA was purchased from Bioneer (Daejeon, Korea). Cells $\left(4.5 \times 10^{5}\right.$ cells in a $100 \mathrm{~mm}$ dish) were transfected with $50 \mathrm{nM}$ of siRNA for 36-48 h using Lipofectamine 2000 reagent (Invitrogen) according to the manufacturer's instructions. The cells were then incubated for $24 \mathrm{~h}$ with serum-free medium and then treated with DHA. The siRNA sequences were as follows: nontargeting control siRNA; $5^{\prime}-A C G$ UGA CAC GUU CGG AGA AUU-3'; Atg5, 5' -AUC CCA UCC AGA GUU GCU UGU GAU C-3'; Atg7, $5^{\prime}$-CCA AGG AUG GUG AAC CUC AGU GAA U-3; and AMPK $5^{\prime}-G G U$ UGG CAA ACA UGA AUU GdTdT-3'.

For the transfection of expression vectors, cells were grown to $70-80 \%$ confluence and then switched to serumfree medium for 2-4h before being transiently transfected 
with HA-tagged myr-Akt1 (Akt-wt) and HA-tagged kinasedead (K179M) dominant negative Aktl (Akt-dd) (kindly provided by Dr. Incheol Shin; Hanyang University, Korea) using Lipofectamine 2000 reagent (Invitrogen) according to the manufacturer's instructions. After $12-18 \mathrm{~h}$, the cells were subjected to different treatments. Control transfections were carried out using an empty pcDNA3 vector.

2.8. Tumor Xenograft Study. Transgenic Fat-1 C57BL/6 mice were provided by Dr. J. X. Kang (Harvard University, USA). Control C57BL/6 mice were purchased from Central Lab Animal Inc. (Seoul, Korea). The control and Fat-1 C57BL/6 mice were kept under specific pathogen-free conditions and received care according to the guidelines of the Institutional Animal Care and Use Committee of Chungnam National University. The Fat-1 C57BL/ 6 mice $(n=5)$ used in this study were heterozygous, male, and 6 weeks old at the time of the experiments. Each mouse was subcutaneously injected with Lewis lung cancer (LLC) cells $\left(3 \times 10^{6}\right.$ in $100 \mu \mathrm{L}$ serum-free DMEM) on day 0 . The tumor size was measured every other day using calipers for 10 days. Tumor size was calculated as length $\times$ wide and volume was calculated as $0.5 \times$ length $\times$ $(\text { wide })^{2}$.

2.9. Immunohistochemistry. After deparaffinization and antigen retrieval, the implanted tumor cell tissues were blocked with Dako protein block (Dako, Glostrup, Denmark); stained with rabbit anti-phospho-Akt (Ser473; 1:250), rabbit antiphospho-AMPK $(1: 250)$, and rabbit anti-LC3B $(1: 250)$ primary antibodies followed by TRITC-conjugated anti-rabbit IgG (1:500); and then counterstained with DAPI. Stained tissues were examined under an Olympus iX70 fluorescence microscope using the DP Controller software. Images from two separate channels were merged.

2.10. Statistical Analysis. Statistical analyses were done as recommended by an independent analyst. These included the unpaired Student's $t$-test. All values are expressed as mean \pm $\mathrm{SD}$, and statistical significance was accepted for $P$ values of $<0.05 .^{*}{ }^{* *}$, and ${ }^{* * *}$ mean $P<0.05, P<0.01$, and $P<0.001$, respectively.

\section{Results and Discussion}

3.1. DHA Reduces Cell Viability and Induces Apoptosis in Human NSCLC Cells. It has been shown previously that DHA induces apoptotic cell death in several types of cancer cells [27, 30-32]. To examine whether DHA induces apoptosis in human NSCLC cells, we tested the effect of DHA on the viability of A549 and H1299 cells in vitro. DHA reduced the viability of both cell lines (Figure 1(a), upper). Moreover, when observed under a light microscope, DHA-treated cells appeared shrunken, rounded, and detached from the culture dishes (Figure 1(a), lower), characteristics suggestive of apoptosis.

To investigate whether this observed DHA-induced reduction in cell viability was actually due to apoptosis, we first examined the cleavage of PARP, an apoptotic marker.
DHA treatment led to an increase in the levels of cleaved PARP in both A549 and H1299 cells (Figure 1(b)). Furthermore, DHA treatment caused a marked increase in the number of Annexin V-positive cells (Figure 1(c)), which is another early apoptotic indicator [33]. We next performed a TUNEL assay and cell-cycle analysis to look for nuclear DNAstrand breaks and hypodiploid DNA formation because both are increased during apoptosis [34]. DHA treatment increased the number of TUNEL-positive A549 cells (Figure 1(d)) and the percentage of A549 cells in the sub-G1 phase (Figure 1(e)). Taken together, these data indicate that DHA induces apoptosis in NSCLC cells.

3.2. DHA Triggers Autophagy as a Prerequisite for Apoptotic Cell Death. Apoptosis and autophagy are highly interconnected [35], and our previous studies showed that DHA activates both of these cascades simultaneously in cervical and prostate cancer cells [23, 24]. To determine whether autophagy is also involved in DHA-induced NSCLC cell death, we initially measured the expression LC3-II (the membrane-bound lipidated form of LC3), a biomarker of autophagic initiation [36]. Western blot analysis revealed that DHA caused a remarkable increase in LC3-II expression (Figure 2(a)). Similarly, DHA led to a dose-dependent increase in the number of GFP-LC3 puncta per virally infected A549 cell (Figure 2(b)). It is known that during autophagy, autophagosomes fuse with lysosomes in which both LC3-II and the cargo are degraded [23]. To examine whether DHA interferes with autophagic flux, we therefore next examined the colocalization of lipidated LC3-II with lysosomes using Lysotracker dye. DHA treatment led to a marked increase in the colocalization of lipidated LC3-II with Lysotracker compared with that in control cells (Figure 2(c)), suggesting that DHA does not block autophagosome-lysosome fusion. To further confirm this, A549 cells were exposed to DHA in the absence or presence of CQ and Bafilomycin A1 (inhibitors of lysosomal acidification) and LC3-II levels were analyzed by immunoblotting (Figure $2(\mathrm{~d})$ ). We found that the DHAinduced increase in LC3-II expression was further increased by CQ and Bafilomycin A1. Taken together, these results indicate that autophagy is activated and may play a role in DHA-induced cell death.

To unveil the relationship between DHA-autophagy and DHA-apoptotic cell death, we used siRNAs to knock down two essential autophagy gene products, Atg5 and Atg7. Although silencing of Atg5 and Atg7 had no effect on the viability of A549 cells, it strongly suppressed DHA-induced autophagy and apoptosis as evidenced by a reduction in LC3II and viability (Figures 3(a) and 3(b)). These results imply that autophagy, at least partially, contributes to DHA-induced apoptotic cell death.

Autophagy has been shown to be essential for cell survival under certain stressful conditions. For example, hypoxia and the anticancer drug, Nelfinavir, induce autophagy by inactivating the growth factor receptors and by suppressing Akt signaling $[37,38]$, both of which play a positive role in cell survival; however, our data indicate that DHA induces autophagy, which enhances cell death. This observation is 

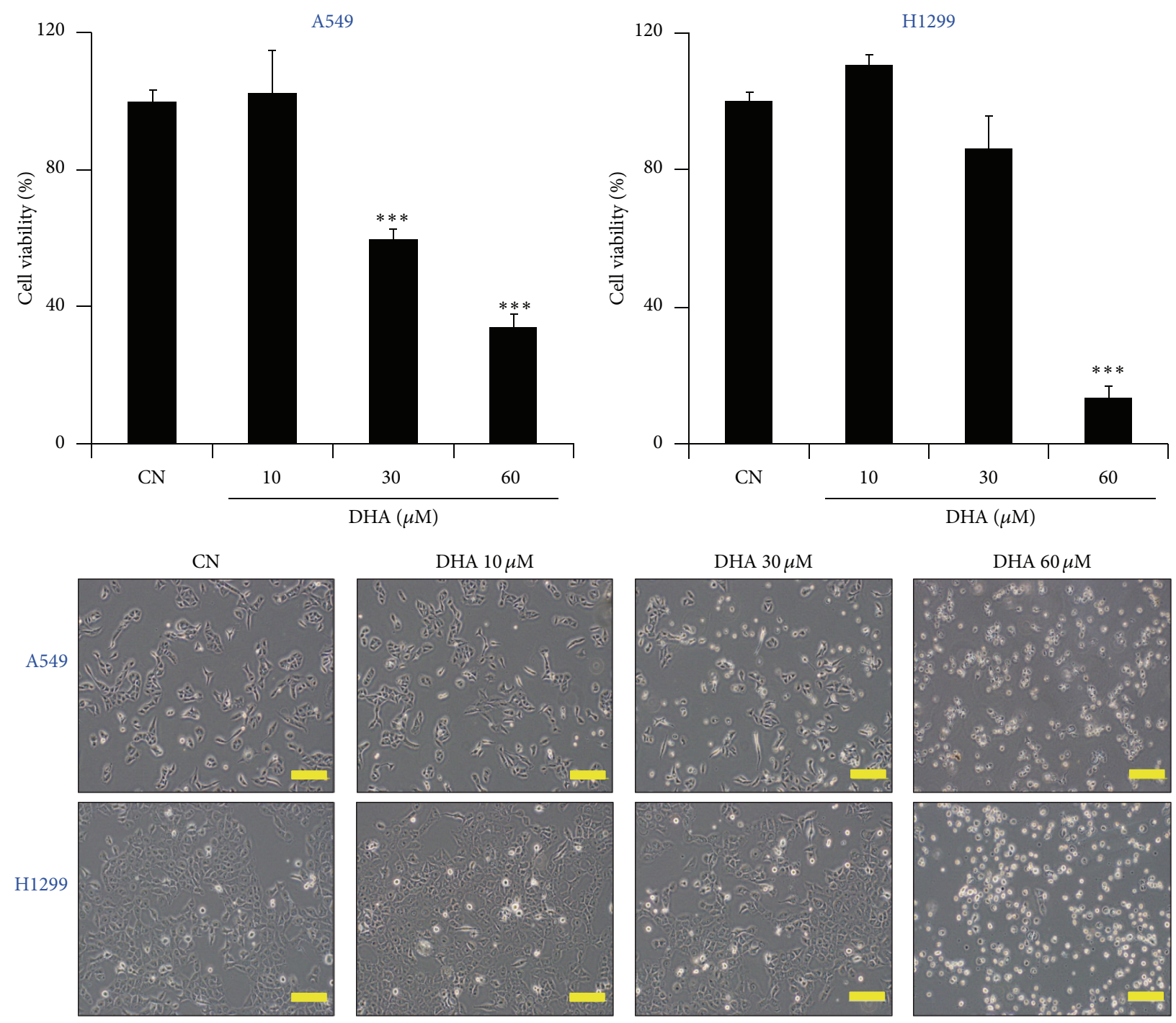

(a)

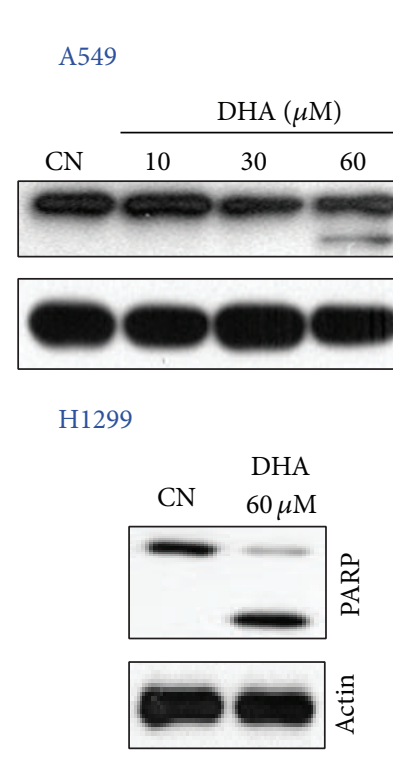

(b)
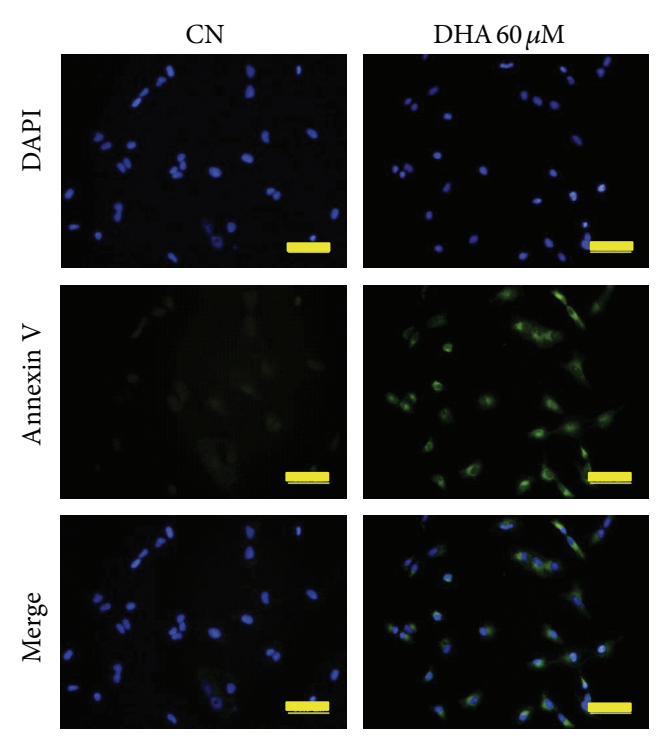

(c)

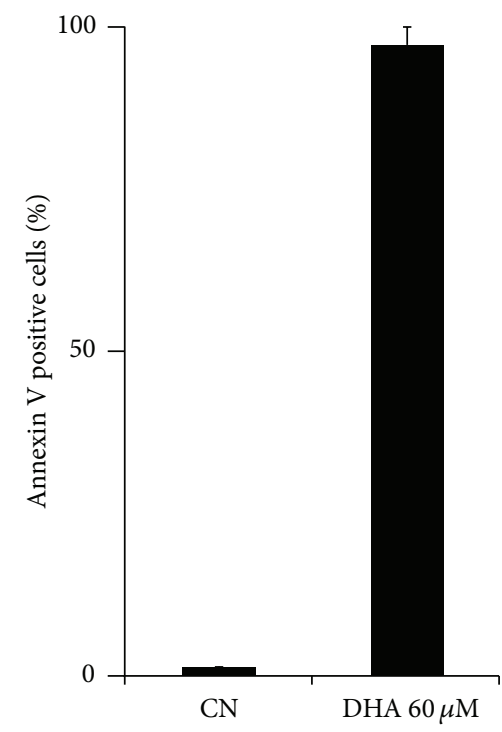

Figure 1: Continued. 

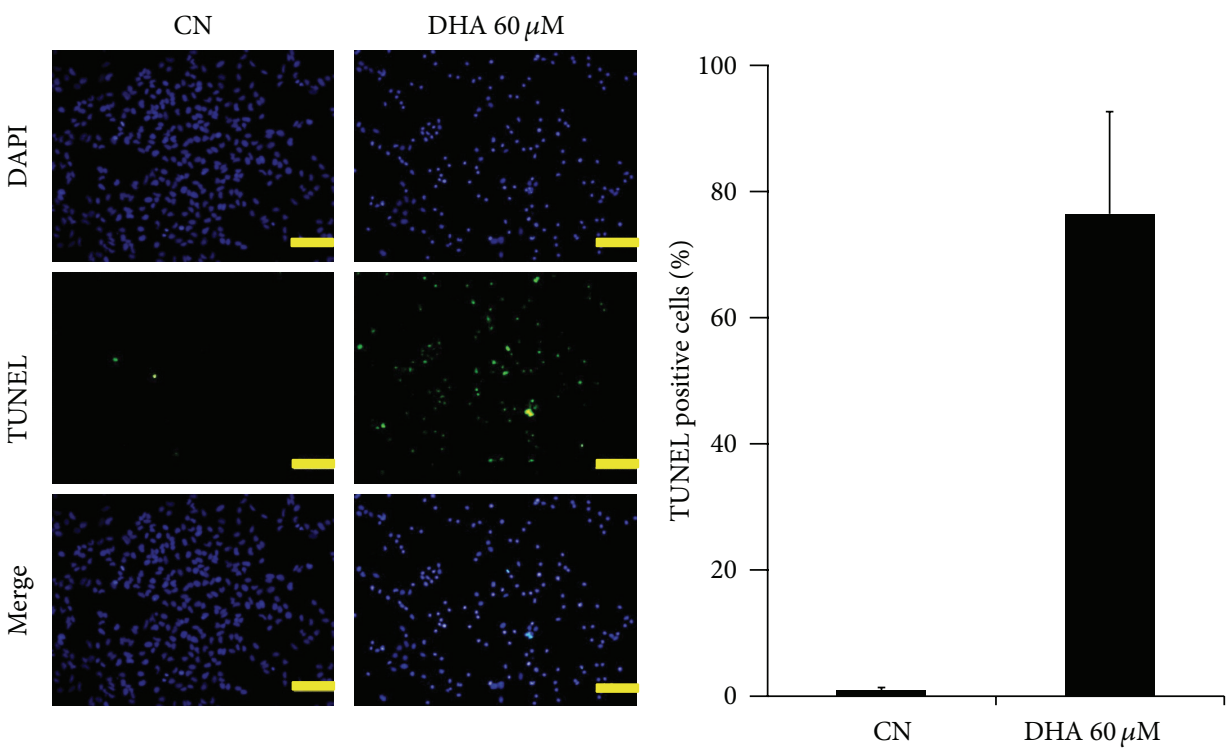

(d)
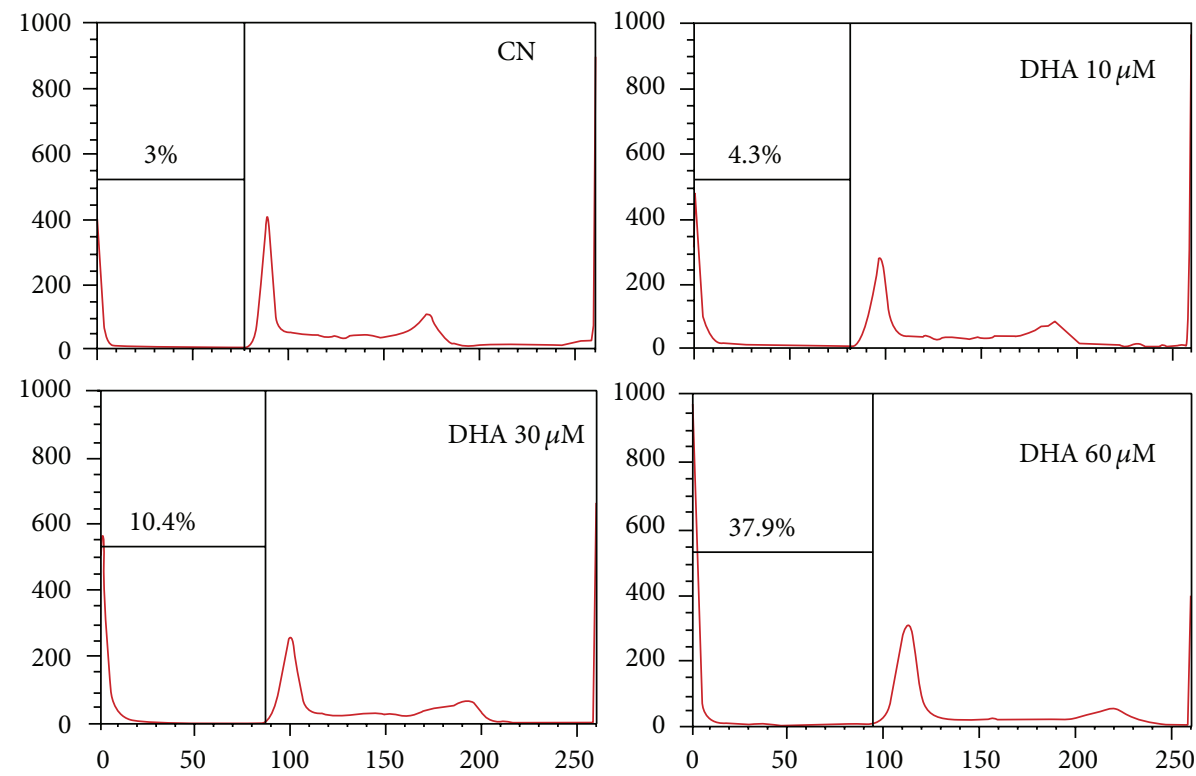

(k)

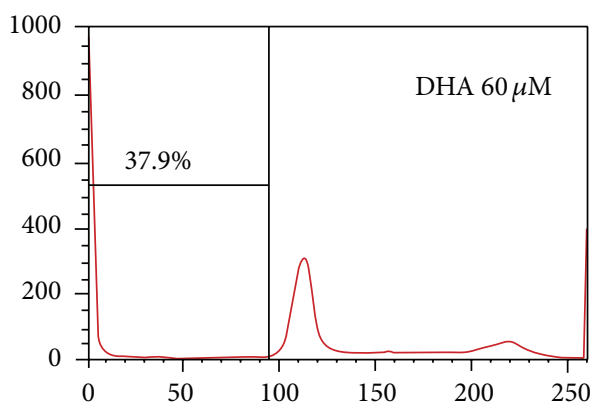

(k)

(e)

FIGURE 1: DHA inhibits cell viability and induces apoptosis in human cancer cells. (a) Upper panel: DHA reduces the viability of A549 and H1299 cells in a dose-dependent manner. Cells were exposed to the indicated doses of DHA for $24 \mathrm{~h}$ and the cell viability was measured in an MTT assay. Each bar represents the mean of three determinations. Each experiment was repeated three times. ${ }^{* * *} P<0.001$. Lower panel: representative images of A549 and H1299 cells treated with DHA for $24 \mathrm{~h}$ (scale bar: $200 \mu \mathrm{m}$ ). (b) DHA induces apoptosis. A549 (upper panel) and H1299 (lower panel) cells were incubated with the indicated doses of DHA for $24 \mathrm{~h}$. The cells were then harvested and western blot analysis was performed with anti-PARP and anti-actin antibodies. (c) Left panel: evaluation of apoptosis by Annexin V staining. Green staining represents Annexin V-positive (apoptotic) cells (scale bar: $50 \mu \mathrm{m}$ ). Right panel: unfixed A549 cells were treated with FITC-Annexin $\mathrm{V}$ and then subjected to flow cytometry to examine changes in the plasma membrane. (d) DHA increases the number of TUNEL-positive cells. A549 cells were plated in a 12-well plate containing glass coverslips and then treated with $60 \mu \mathrm{M}$ DHA for $6 \mathrm{~h}$. Following treatment, apoptosis was detected using the DeadEnd Fluorometric TUNEL System. Left panel: representative fluorescence microscopy images (scale bar: $200 \mu \mathrm{m}$ ). Right panel: the percentage of TUNEL-positive cells in the presence or absence of DHA was expressed relative to the total number of DAPI-stained nuclei. TUNEL-positive cells were counted in three different fields and the numbers averaged. (e) DHA increases the number of NSCLC cells in the sub-G G $_{1}$ phe. A549 cells were seeded and treated with the indicated doses of DHA for $24 \mathrm{~h}$. The cell-cycle distribution of DHA-treated cells was analyzed by flow cytometry as described in Section 2. Data were analyzed using FlowJo software. 
A549 DHA $(\mu \mathrm{M})$

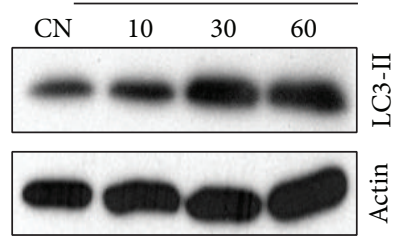

H1299

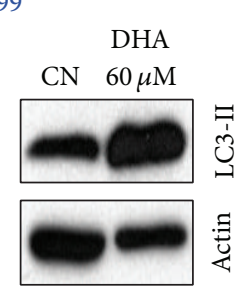

(a)
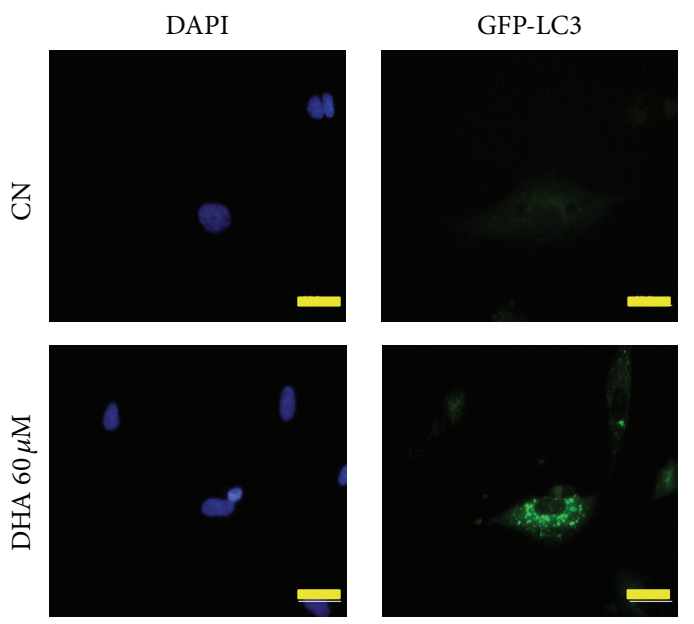

(c)

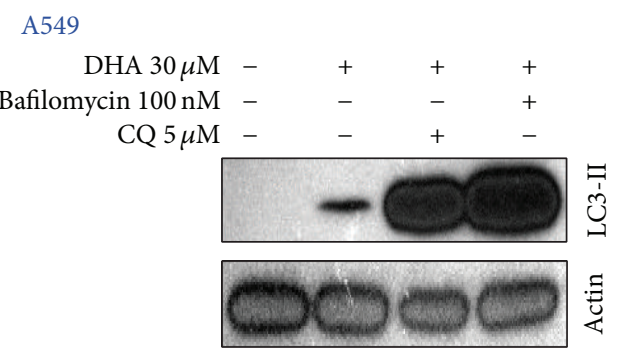

(d)

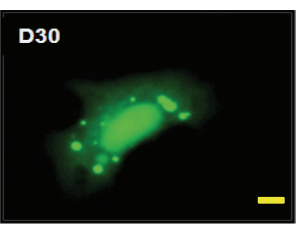

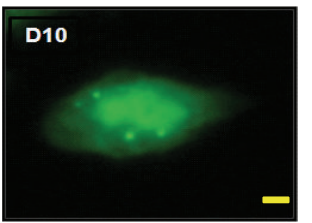
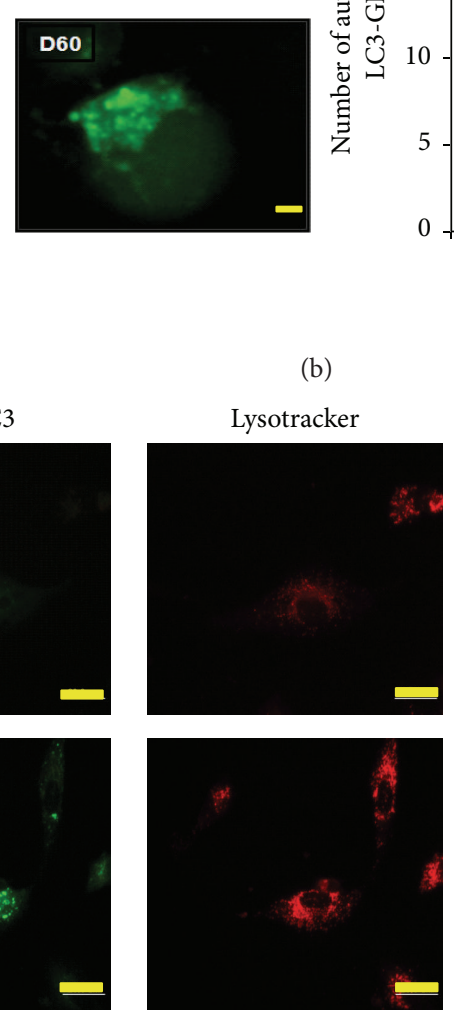

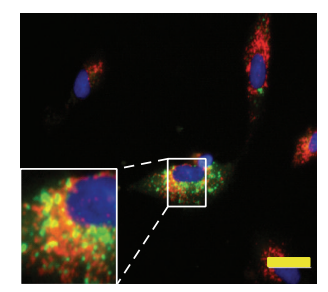

H1299

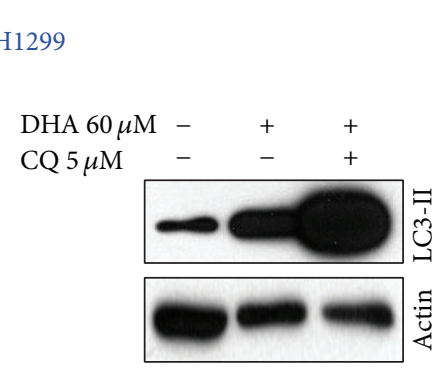

(b)
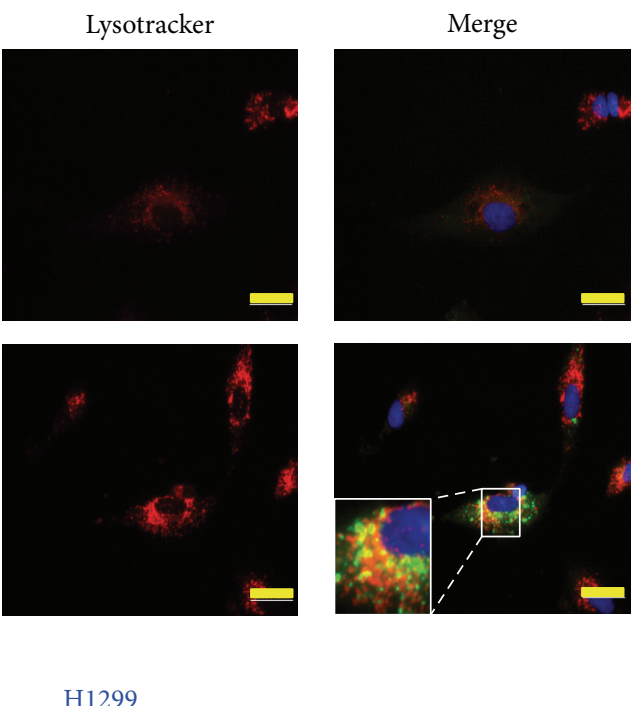

FIGURE 2: DHA induces autophagy. (a) DHA increased the expression of LC3-II in a dose-dependent manner. A549 (upper panel) and H1299 (lower panel) cells were exposed to the indicated doses of DHA for $24 \mathrm{~h}$. Protein lysates were then prepared, separated in SDS polyacrylamide gels, and immunoblotted with antibodies against LC3-II and actin. (b) Formation of GFP-LC3 puncta in DHA-treated NSCLC cells. A549 cells were transfected with a GFP-LC3 plasmid and then exposed to the indicated doses of DHA for another $24 \mathrm{~h}$ before counterstaining with DAPI. Left panel: representative fluorescence microscopy images are shown (scale bar: $2 \mu \mathrm{m}$ ). Right: the number of autophagosomes was quantified as the number of GFP-LC3 puncta per transfected cell. Data are expressed as the mean \pm SD of ten determinations (each in two separate experiments). ${ }^{* * *} P<0.001$. (c) DHA activates autophagic flux in NSCLC cells. Cells were transfected with the GFP-LC3 expression vector using Lipofectamine 2000 reagent for $17 \mathrm{~h}$ and then treated with $60 \mu \mathrm{M}$ DHA for another $4 \mathrm{~h}$. DHA-treated cells were then stained with Lysotracker dye. Representative fluorescence microscopy images are shown. Data are expressed as the mean \pm SD of five determinations (each in three separate experiments) (scale bar: $10 \mu \mathrm{m}$ ). (d) DHA-induced autophagy increases NSCLC cell death. A549 (left) and H1299 (right) cells were incubated for $1 \mathrm{~h}$ in the presence or absence of the indicated doses of Bafilomycin and CQ before incubation with indicated doses of DHA for $24 \mathrm{~h}$. Cell lysates were prepared and examined by western blotting. 


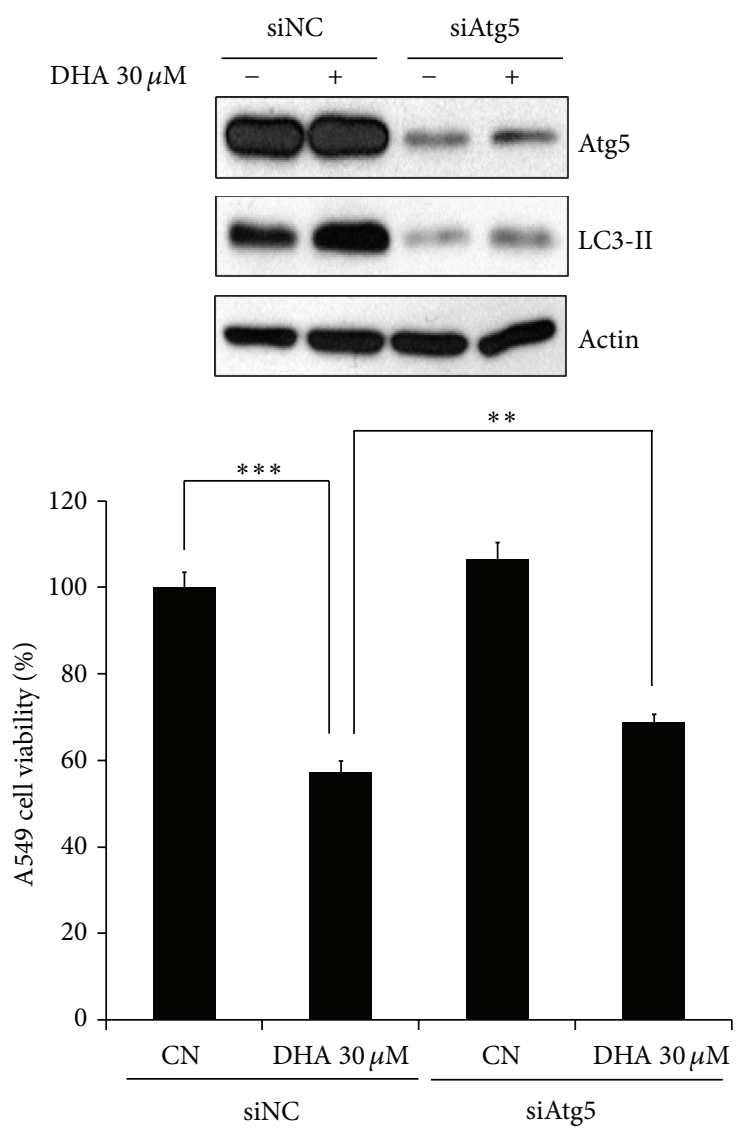

(a)

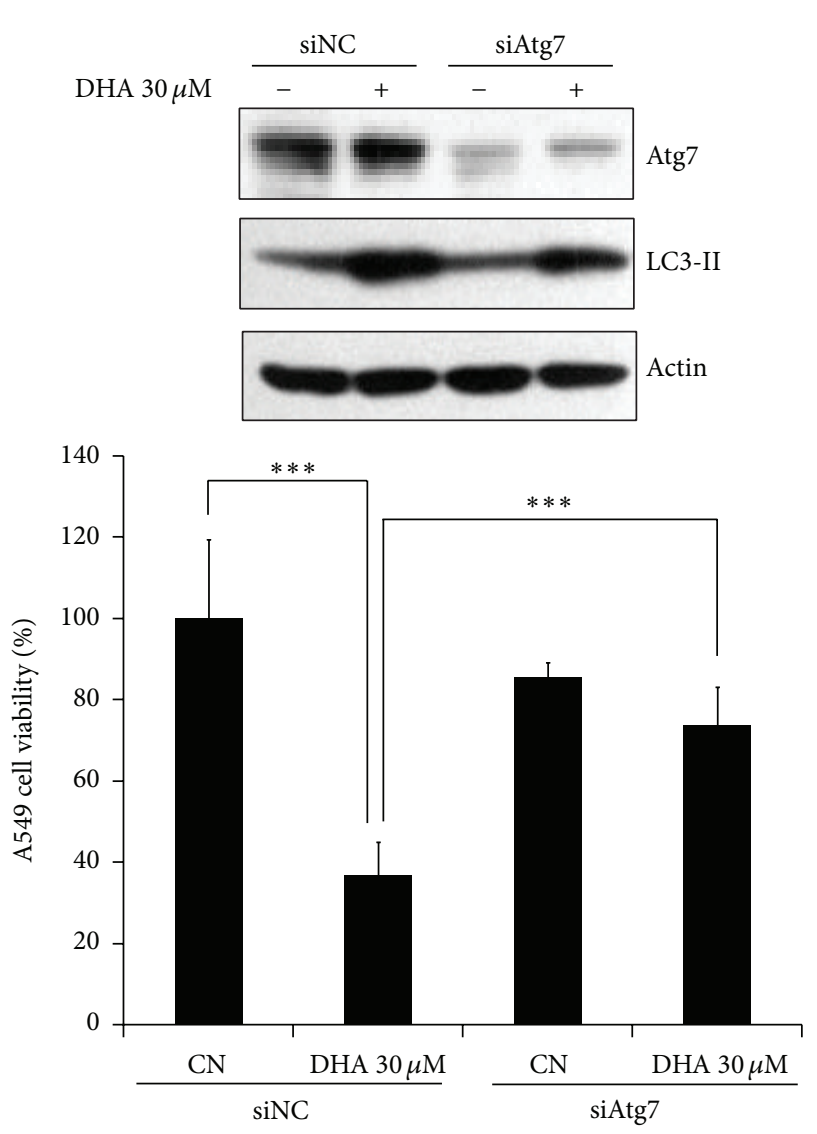

(b)

FIGURE 3: DHA-induced autophagy is required for apoptotic cell death. The indicated cancer cell lines were treated with nontargeting control small interfering RNA (siNC) or siRNAs specific for autophagy-related Atg5 (a) and Atg7 (b). At $18 \mathrm{~h}$ after transfection, cells were incubated with the indicated doses of DHA for $24 \mathrm{~h}$. Next, cells were harvested and subjected to western blot analysis with the following antibodies: Atg5, Atg7, LC3-II, and actin (upper panel). Cell viability was measured in an MTT assay (lower panel). ${ }^{* *} P<0.01$ and ${ }^{* * *} P<0.001$. Data are representative of three independent experiments, all with similar results.

in line with other reports showing that Clioquinol and Rhabdastrellic acid-A promote cell death in hepatocellular carcinoma, lung adenocarcinoma, myeloma, and leukemia cells lines by inducing autophagy [36, 39]. Although the exact mechanism by which autophagy contributes to DHAinduced apoptosis in NSCLC cells is still unclear, it is known that autophagy can promote cell death by selectively eliminating vital components, such as mitochondria and peroxisomes [40]. We have reported that DHA-induced apoptosis is associated with mitochondrial damage [24, 31]; therefore, it is possible that DHA-induced autophagy is triggered by eliminating components that are essential for survival, such as mitochondria.

3.3. DHA-Mediated Downregulation of mTOR Signaling Is Associated with Autophagy Induction. mTOR is the key negative regulator of autophagy [4]. To examine whether mTOR inhibition is involved in DHA-induced autophagy, we investigated the expression of mTOR signaling-related molecules in NSCLC cells after DHA treatment. DHA reduced the levels of phospho-mTOR in both A549 (Figure 4(a), left panel) and H1299 (Figure 4(a), right panel) cells, indicating that the activity of mTOR is repressed by DHA. Consistent with this, the levels of mTOR's two readout molecules, phospho-S6K1 and phospho-4E-BP1, were also found to be decreased in A549 cells. Meanwhile, increases in p27 (whose activation is indicative of mTOR inhibition) [23] were observed in DHAtreated A549 and H1299 cells (Figure 4(a)), suggesting that DHA indeed suppresses the mTOR signaling pathway. Next, to confirm the role of mTOR in DHA-induced cell death, we pretreated A549 cells with rapamycin followed by DHA. We found that DHA-induced decreases in uncleaved PARP and increases in LC3-II expression in A549 cells were enhanced by pretreatment with rapamycin (Figure 4(b)). These results imply that DHA-induced autophagy and apoptosis are associated with $\mathrm{mTOR}$ inhibition.

mTOR is directly linked to PI3K/Akt signaling [41], and the PI3K/Akt/mTOR signaling pathway plays an important role in cell proliferation and survival [42]. We then asked whether PI3K/Akt is associated with DHA-induced mTOR 


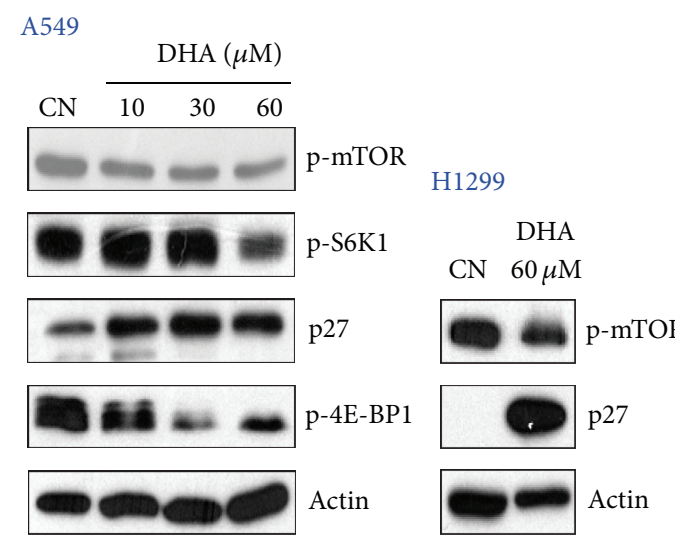

(a)

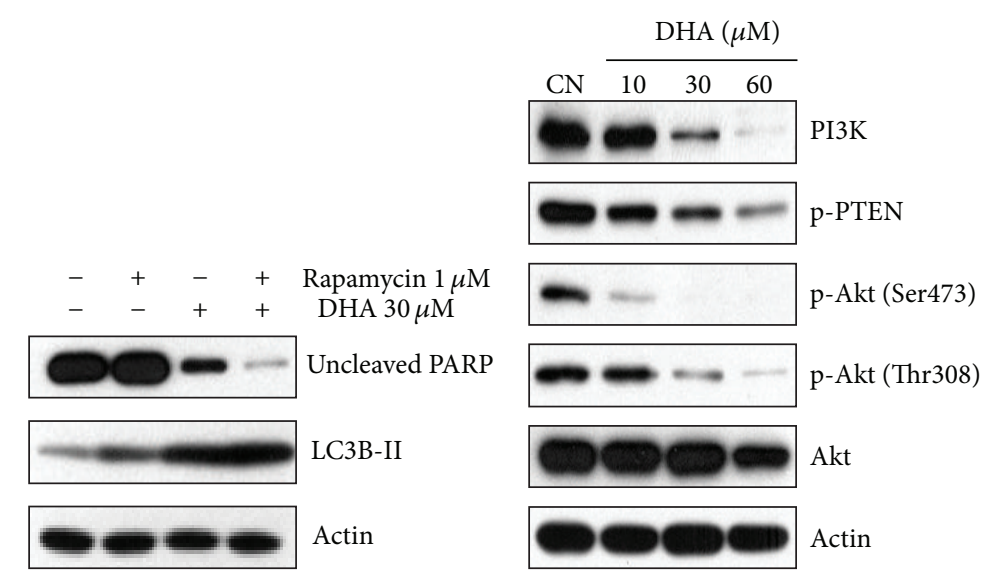

(b) (c)

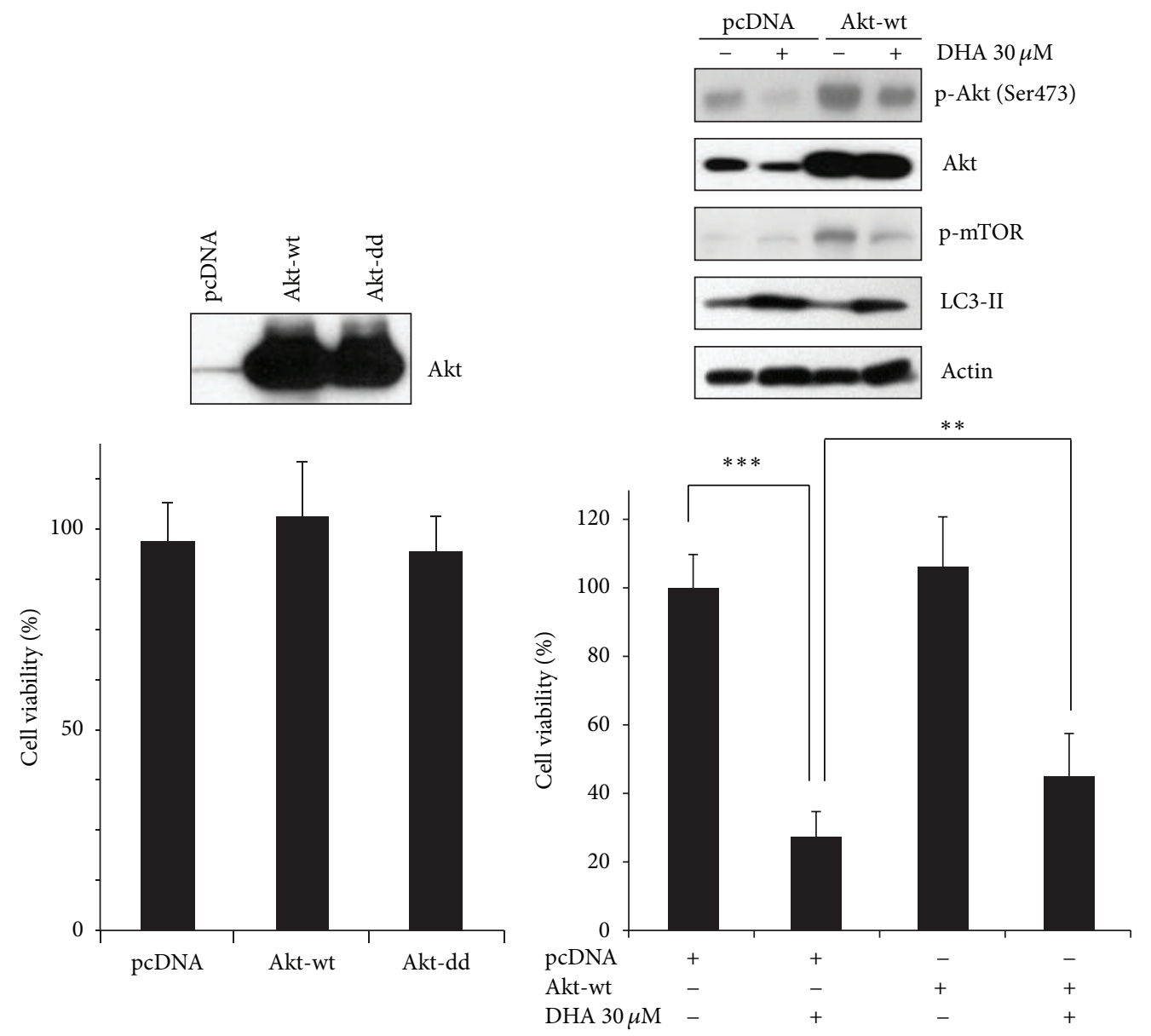

(d)

(e)

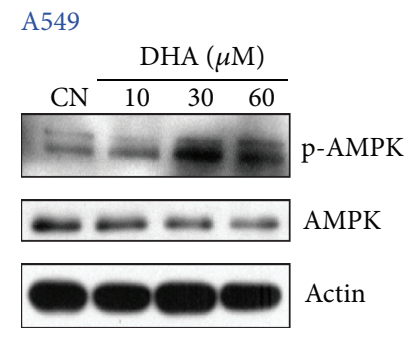

H1299

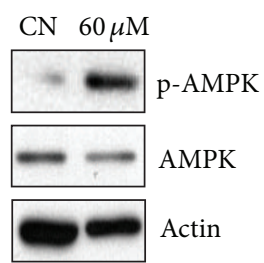

(f)

Figure 4: Continued. 


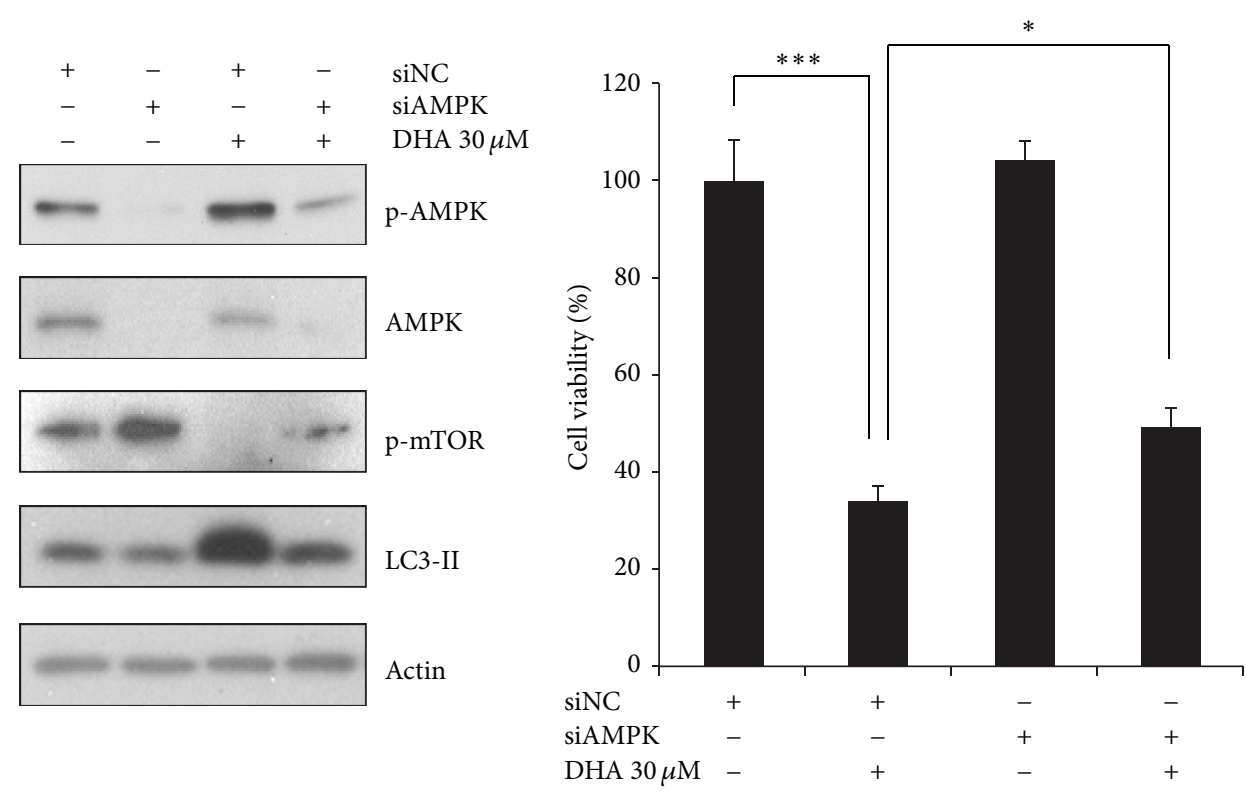

(g)

FIGURE 4: DHA-mediated downregulation of mTOR signaling is related to the induction of autophagy. (a) DHA downregulated mTOR signaling in a dose-dependent manner. A549 (left panel) and H1299 (right panel) cells were incubated with the indicated doses of DHA for $24 \mathrm{~h}$ and then subjected to western blot analysis with antibodies against phospho-mTOR, phospho-S6K1, p27, 4E-BP1, and actin. (b) Rapamycin accelerated autophagy and cell death by inhibiting mTOR. A549 cells were incubated for $1 \mathrm{~h}$ with or without $1 \mu \mathrm{M}$ rapamycin before incubation for $24 \mathrm{~h}$ with $30 \mu \mathrm{M}$ DHA. Cell lysates were prepared and examined by western blotting. (c) DHA reduces PI3K/Akt signaling pathway. Western blotting with antibodies against phosphatidylinositol 3-kinase (PI3K)/Akt signaling molecules showed that DHA downregulates PI3K/Akt signaling in a dose-dependent manner. (d)-(e) Expression of Akt-wt partially rescued DHA-induced NSCLC cell death. pcDNA and a Akt-wt vector were transfected into cells using Lipofectamine 2000 reagent for $12 \mathrm{~h}$. The cells were then exposed to $30 \mu \mathrm{M}$ for another $24 \mathrm{~h}$. Cell viability was examined in an MTT assay ((d) and (e), lower panel) and the cell lysates were analyzed by western blotting with antibodies against phospho-Akt, Akt, phospho-mTOR, and actin ((e), upper panel). ${ }^{* * *} P<0.001$. (f) DHA treatment led to a dose-dependent increase in phospho-AMPK levels. A549 (left panel) and H1299 (right panel) cells were treated with indicated doses of DHA for $24 \mathrm{~h}$ and cell lysates were examined by western blotting. (g) siAMPK reduced DHA-induced autophagy and inhibited cell death in NSCLC cells by upregulating mTOR signaling. A549 cells were transfected with a siNC or siAMPK and then exposed to $30 \mu \mathrm{M}$ DHA for $24 \mathrm{~h}$. Left panel: western blot analysis of phospho-AMPK, AMPK, phospho-mTOR, LC3-II, and actin expression. Right panel: cell viability was measured in an MTT assay. ${ }^{*} P<0.05$ and ${ }^{* * *} P<0.001$.

inactivation. To test this, we examined the expression of PI3K/Akt signaling molecules and found that DHA induced a marked reduction in PI3K, Akt, and phospho-PTEN (a negative regulator of the PI3K) (Figure 4(c)). Next, to obtain evidence for the interconnection between decreased PI3K/Akt signaling and DHA-induced cell death, we overexpressed Akt in A549 cells before DHA treatment (overexpression in itself had no significant effect on the cell viability of A549 cells) (Figure 4(d)). DHA treatment led to a reduction in cell viability and increased the levels of phosphomTOR and LC3-II; however, these phenomena were partially reversed by Akt overexpression (Figure 4(e)). These data suggest that DHA-induced cell death is also associated with Akt inhibition in NSCLC cells. Evidence suggests that DHA can disrupt the association between lipid rafts and epidermal growth factor receptor (EGFR), leading to inactivation of EGFR and its downstream PI3K/Akt signaling in lung cancer cells [43]. Accordingly, it is reasonable to speculate that DNA might inhibit the PI3K/Akt signaling pathway by disrupting EGFR phosphorylation and its association with lipid rafts; however, further studies are needed to investigate whether this is the mechanism underlying DHA-mediated Akt inactivation.

In addition to Akt, another key mediator of mTOR is AMPK, which has been demonstrated to negatively regulate mTOR activity [15]. We sought subsequently to determine whether DHA-induced mTOR inactivation involves AMPK in NSCLC cells. To this end, we first measured the levels of AMPK in DHA-treatment A549 and H1299 cells. DHA treatment led to increased AMPK activity in both A549 and H1299 cells, as evidenced by the promoted expression levels of phospho-AMPK (Figure 4(f)). Importantly, knockdown of AMPK not only rescued DHA-induced mTOR inactivation, but also inhibited DHA-induced autophagy and cell death in A549 cells (Figure 4(g)). These results demonstrated that DHA-induced autophagy mediated by mTOR inhibition, at least partly, attributes to AMPK activation. Previously, endoplasmic reticulum (ER) stress has been shown to elevate AMPK levels, leading to the upregulation of $\mathrm{Ca}^{2+}-\mathrm{CaMKK} \beta$ signaling and autophagy-related genes such as Atg5 and Atg12 [44]. We found that the levels of phosphoeukaryotic translation initiation factor- $2 \alpha$, which reflects ER stress, 
Wild-type mouse
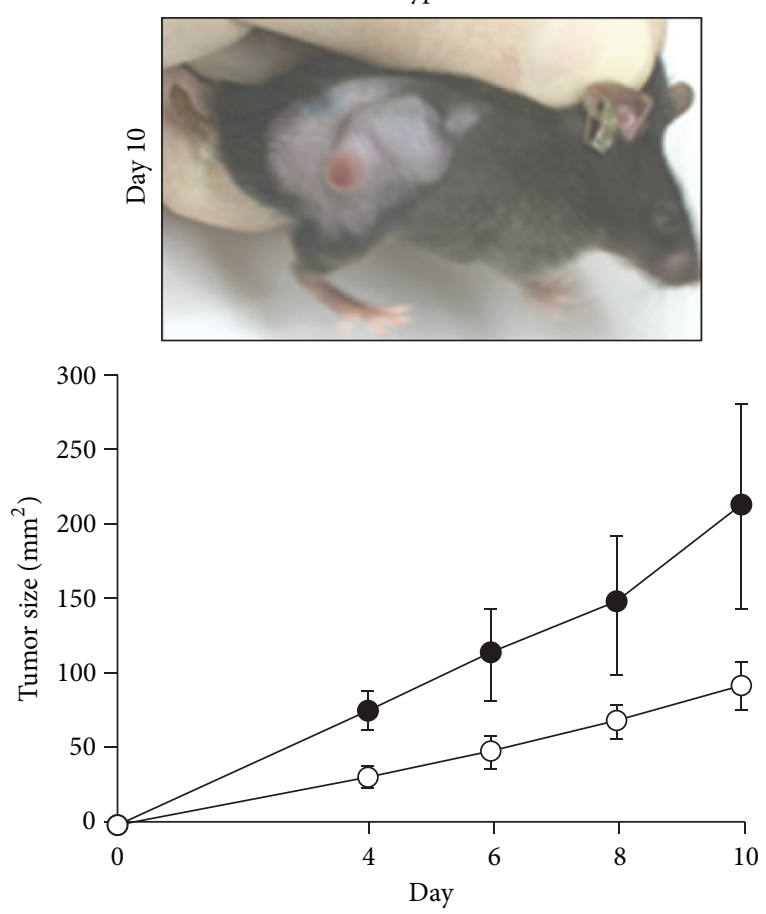

- Wild type

- Fat-1

Wild-type mouse

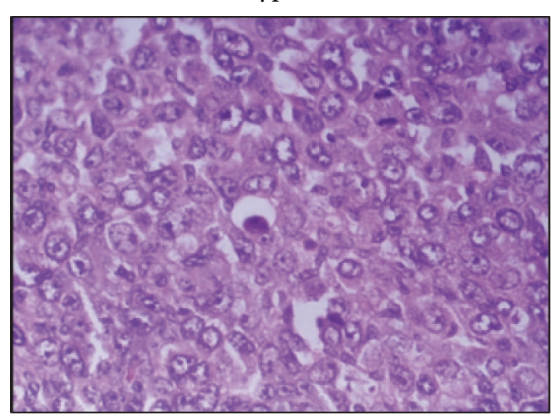

DAPI
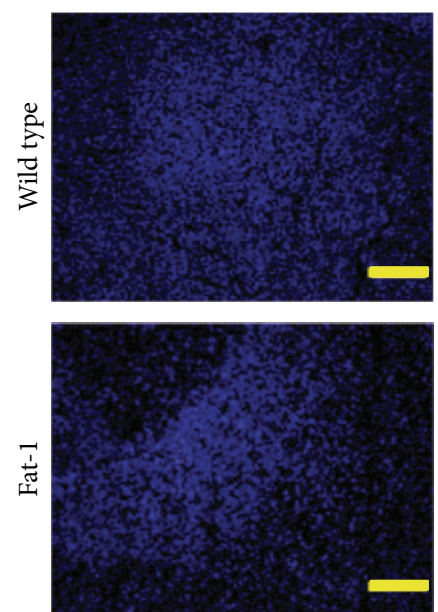

(a)

TUNEL
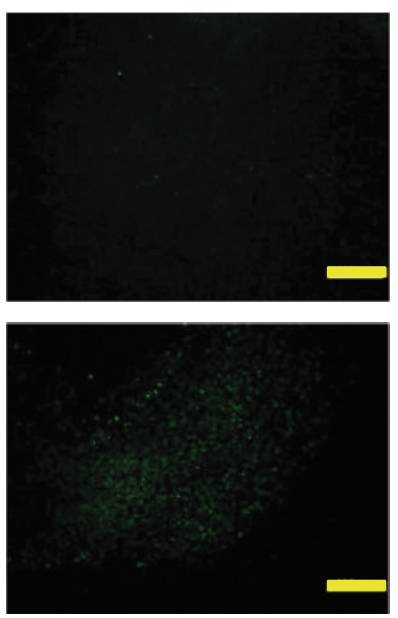

(b)
Fat-1 mouse
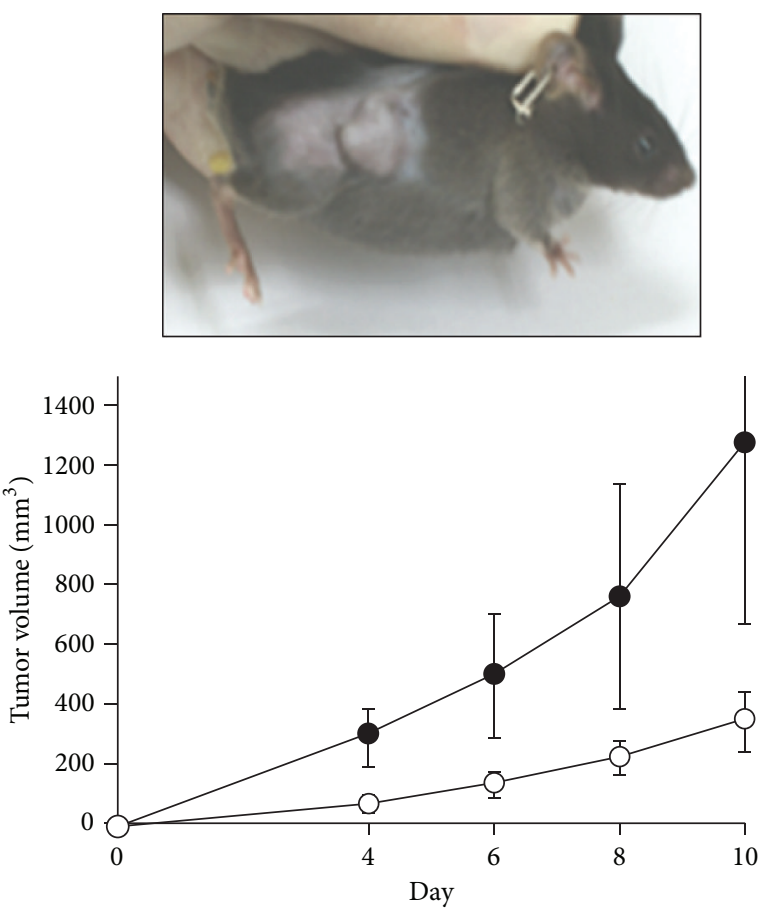

- Wild type
-O- Fat-1

Fat-1 mouse

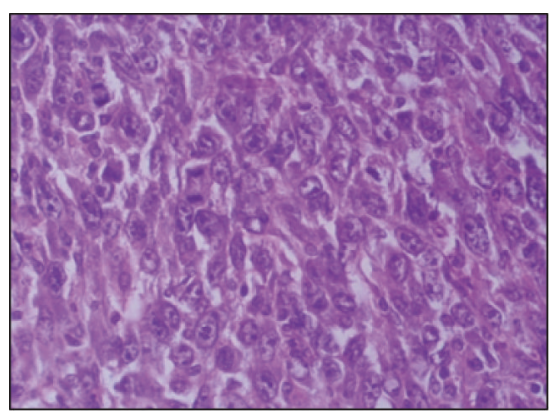

Merge
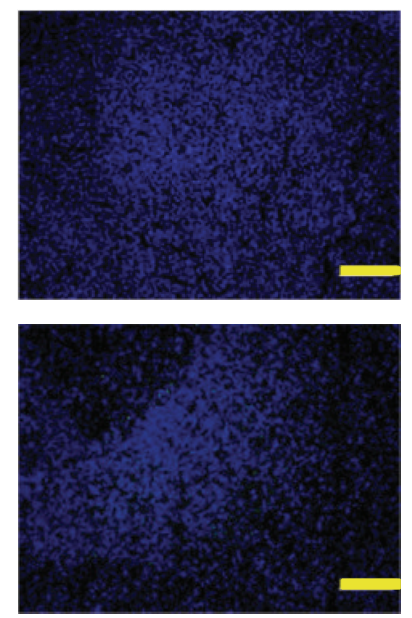

Figure 5: Continued. 

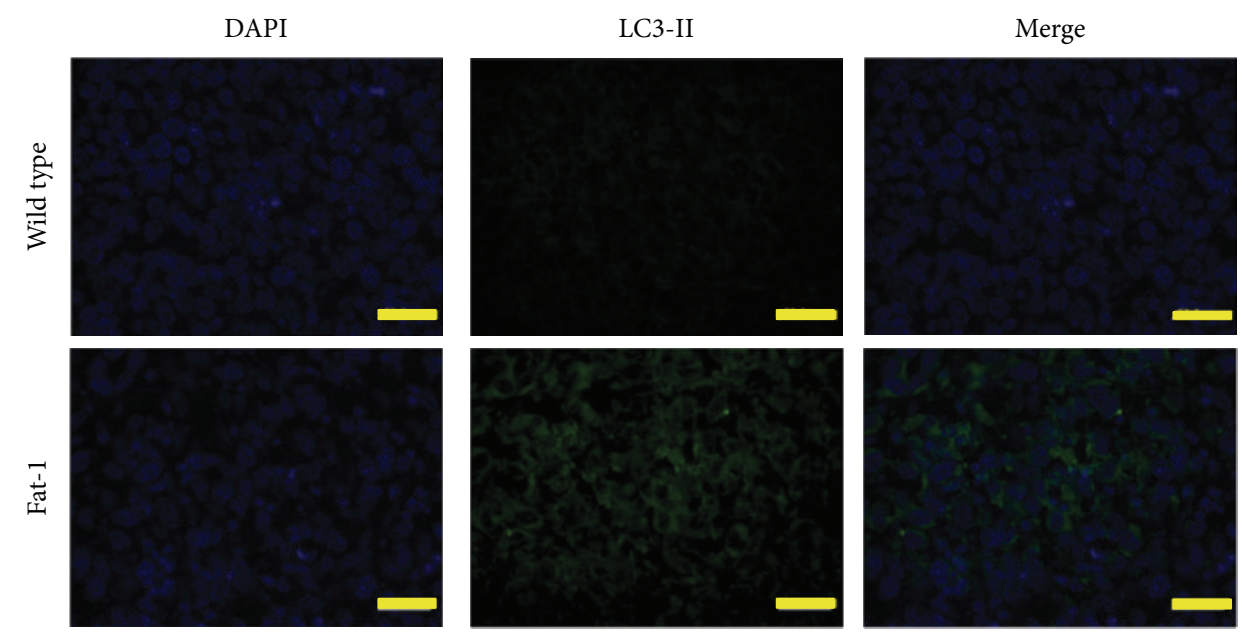

(c)

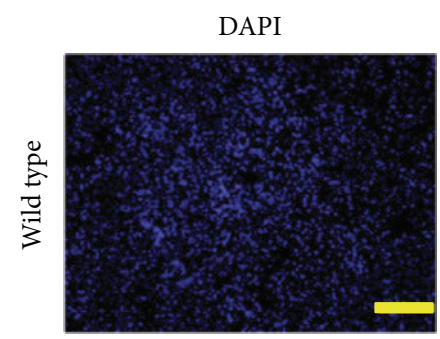

p-Akt (Ser473)

Merge
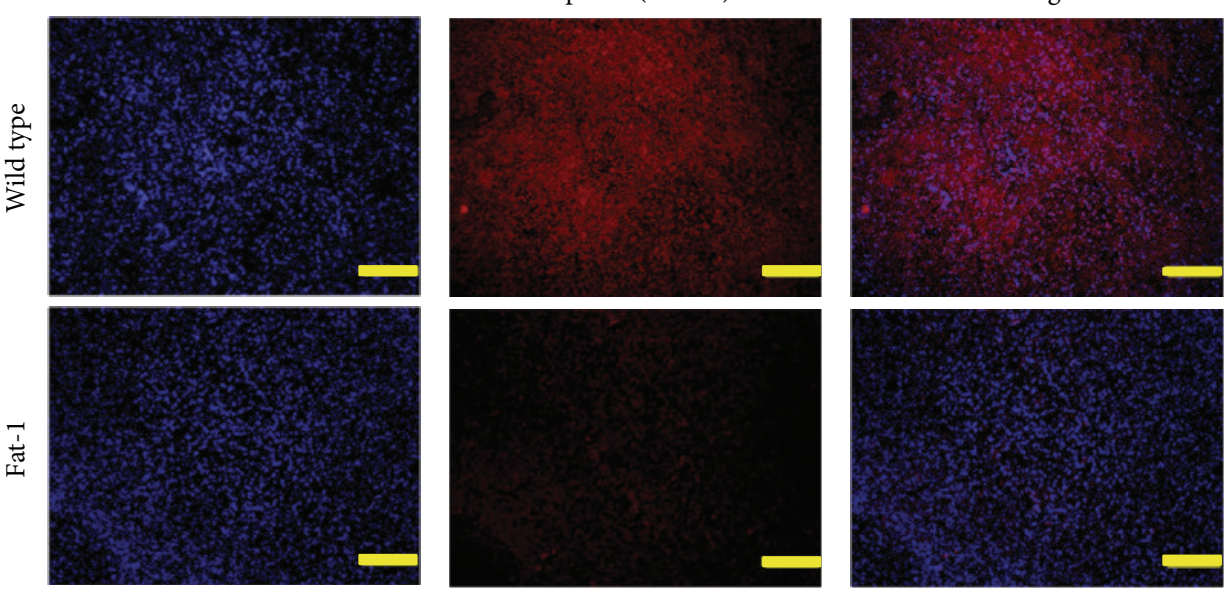

(d)
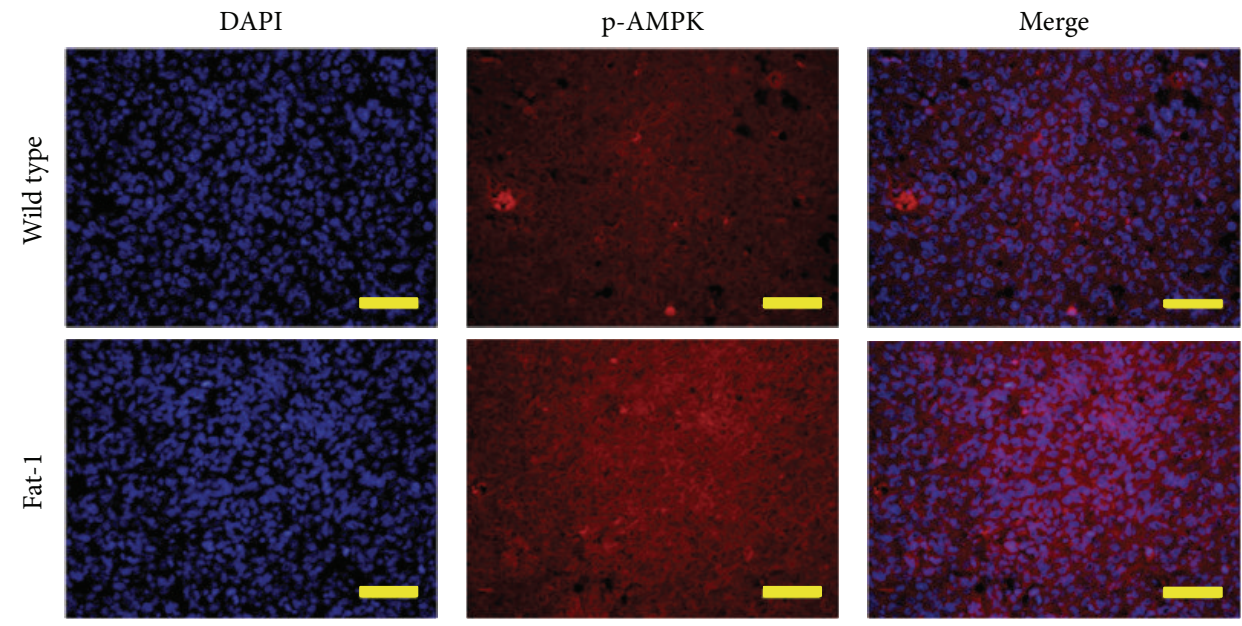

(e)

FIGURE 5: $\omega 3$-PUFAs suppress tumor growth in vivo by inhibiting phospho-Akt and phospho-AMPK, thereby inducing apoptosis and autophagy. (a) Effect of $\omega 3$-PUFA on tumorigenicity. Upper panel: LLC cells $\left(3 \times 10^{6}\right.$ cells $)$ were injected subcutaneously into the flanks of wild-type and Fat-1 transgenic mice. Tumor size and volume were monitored every other day for 10 days. Tumor size and volume (middle) were calculated as described in Section 2. Lower panel: hematoxylin and eosin (H\&E) staining. ((b)-(e)) Representative fluorescence images showing the TUNEL assay results (b), LC3-II (c), phospho-Akt (Ser473) (d), and phospho-AMPK (e). Fluorescently stained tissues were observed under a fluorescence microscope using DP Controller software (Olympus) for image acquisition. Scale bars: $200 \mu \mathrm{m}$. 


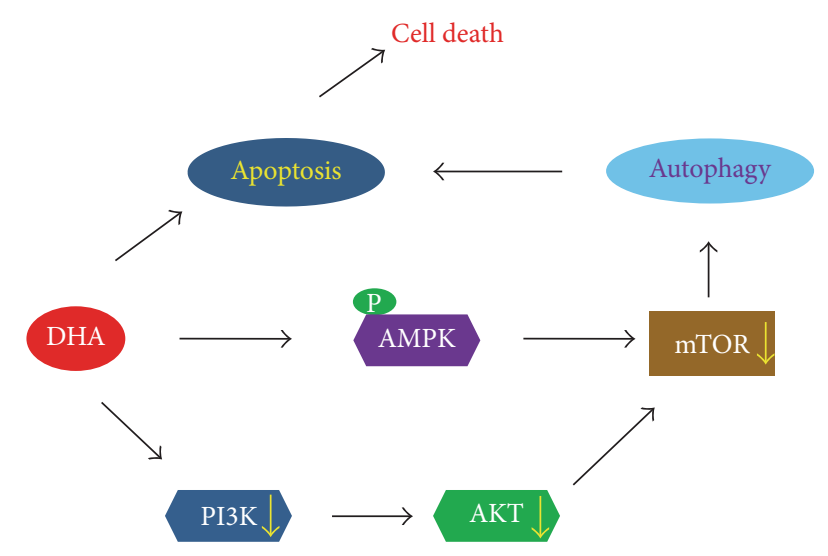

Figure 6: Schematic model of DHA-induced apoptosis and autophagy in NSCLC cells. DHA-induced autophagy and apoptosis in lung cancer cells are triggered by inhibition of mTOR activation via AMPK activation and PI3K/Akt inhibition.

increased in DHA-treated A549 cells in a time-dependent manner (data not shown). Thus, although the exact mechanism underlying DHA-mediated AMPK activation remains unknown, it is possible that AMPK activation in DHA-treated cells is induced by the activation of $\mathrm{Ca}^{2+}-\mathrm{CaMKK} \beta$ and autophagy-related genes via ER stress.

3.4. W3-PUFAs Suppress Tumor Growth In Vivo by Inhibiting the Expression of Phospho-Akt and Phospho-AMPK, Thereby Inducing Apoptosis and Autophagy. The in vitro results presented above show that DHA reduced the viability of human NSCLC cells. We next examined the effect of DHA on tumor formation and growth in Fat-1 transgenic mice. Fat1 transgenic mice express $\omega 3$-desaturase and thus produce higher levels of $\omega 3$-PUFAs than wild-type (WT) mice [45]. The mouse origined NSCLC, LLC cells were subcutaneously injected into WT mice and Fat-1 transgenic mice, and tumor size and volume were measured. We found that both the size and volume of the tumors in Fat-1 transgenic mice were markedly lower than those in WT mice (Figure 5(a)), suggesting that $\omega 3$-PUFAs suppress the growth of NSCLC cells in vivo. To verify whether apoptosis and autophagy played a role in this process, we performed TUNEL assays and indirect immunofluorescence assays on tumor tissue sections to examine apoptosis and autophagy levels, respectively. The number of TUNEL-positive cells and the number of LC3II puncta were higher in Fat-1 tumors than in WT tumors (Figures 5(b) and 5(c)), indicating that both apoptosis and autophagy played a role in inhibiting tumor growth in Fat1 transgenic mice. Next, to determine whether PI3K/Akt and AMPK signaling were responsible for the increased levels of apoptosis and autophagy, we examined the levels of Akt and AMPK in tumor tissues by immunohistochemistry. As shown in Figures 5(d) and 5(e), the levels of phospho-Akt (Ser473) decreased and the levels of phospho-AMPK increased in Fat1 tumor tissues. Together, these data demonstrate that $\omega 3$ PUFAs induce apoptosis and autophagy in vivo by regulating AMPK and PI3K/Akt signaling.

\section{Conclusions}

In conclusion, we describe for the first time that DHA triggers autophagy and apoptosis in NSCLC cells, which simultaneously promotes cell death. Our results indicate that the DHA-induced autophagy and apoptosis are controlled by repressing mTOR through AMPK activation and PI3K/Akt inhibition (Figure 6). These data suggest that DHA may represent a potentially useful reagent for treating human NSCLC in clinical settings.

\section{Conflict of Interests}

The authors declare that there is no conflict of interests regarding the publication of this paper.

\section{Authors' Contribution}

Nayeong Kim and Soyeon Jeong contributed equally to this work.

\section{Acknowledgments}

The authors thank Professor Chang Deok Kim and Dr. Incheol Shin for the gift of GFP-LC3B adenovirus and vectors. They also would like to thank Dr. J. X. Kang for providing them with transgenic Fat-1 C57BL/6 mice. This work was supported by the National Research Foundation of Korea (NRF) Grant funded by the Korea government (MSIP) (20070054932).

\section{References}

[1] R. Siegel, J. Ma, Z. Zou, and A. Jemal, "Cancer statistics, 2014," CA: A Cancer Journal for Clinicians, vol. 64, no. 1, pp. 9-29, 2014.

[2] R. Sundar, R. Soong, B.-C. Cho, J. R. Brahmer, and R. A. Soo, "Immunotherapy in the treatment of non-small cell lung cancer," Lung Cancer, vol. 85, pp. 101-109, 2014.

[3] L. Song, H. Xiong, J. Li et al., "Sphingosine kinase-1 enhances resistance to apoptosis through activation of PI3K/Akt/NFkappaB pathway in human non-small cell lung cancer," Clinical Cancer Research, vol. 17, pp. 1839-1849, 2011.

[4] C. He and D. J. Klionsky, "Regulation mechanisms and signaling pathways of autophagy," Annual Review of Genetics, vol. 43, pp. 67-93, 2009.

[5] N. Mizushima, Y. Ohsumi, and T. Yoshimori, "Autophagosome formation in mammalian cells," Cell Structure and Function, vol. 27, no. 6, pp. 421-429, 2002.

[6] S. A. Tooze and T. Yoshimori, "The origin of the autophagosomal membrane," Nature Cell Biology, vol. 12, no. 9, pp. 831-835, 2010.

[7] N. Mizushima, "Autophagy: process and function," Genes \& Development, vol. 21, no. 22, pp. 2861-2873, 2007.

[8] C. M. Fader and M. I. Colombo, "Autophagy and multivesicular bodies: two closely related partners," Cell Death \& Differentiation, vol. 16, no. 1, pp. 70-78, 2009.

[9] D. Glick, S. Barth, and K. F. Macleod, "Autophagy: cellular and molecular mechanisms," The Journal of Pathology, vol. 221, no. 1, pp. 3-12, 2010. 
[10] S. Faivre, G. Kroemer, and E. Raymond, "Current development of mTOR inhibitors as anticancer agents," Nature Reviews Drug Discovery, vol. 5, no. 8, pp. 671-688, 2006.

[11] M. Laplante and D. M. Sabatini, "mTOR signaling at a glance," Journal of Cell Science, vol. 122, no. 20, pp. 3589-3594, 2009.

[12] M. Laplante and D. M. Sabatini, "mTOR signaling in growth control and disease," Cell, vol. 149, no. 2, pp. 274-293, 2012.

[13] A. Hahn-Windgassen, V. Nogueira, C.-C. Chen, J. E. Skeen, N. Sonenberg, and N. Hay, "Akt activates the mammalian target of rapamycin by regulating cellular ATP level and AMPK activity," The Journal of Biological Chemistry, vol. 280, no. 37, pp. 3208132089, 2005.

[14] D. G. Hardie, "AMP-activated/SNF1 protein kinases: conserved guardians of cellular energy," Nature Reviews Molecular Cell Biology, vol. 8, no. 10, pp. 774-785, 2007.

[15] N. Hay and N. Sonenberg, "Upstream and downstream of mTOR," Genes \& Development, vol. 18, no. 16, pp. 1926-1945, 2004.

[16] R. Nawroth, F. Stellwagen, W. A. Schulz et al., "S6k1 and 4EBP1 are independent regulated and control cellular growth in bladder cancer," PLoS ONE, vol. 6, no. 11, Article ID e27509, 2011.

[17] D. C. Fingar, C. J. Richardson, A. R. Tee, L. Cheatham, C. Tsou, and J. Blenis, "mTOR controls cell cycle progression through its cell growth effectors $\mathrm{S} 6 \mathrm{~K} 1$ and 4E-BP1/eukaryotic translation initiation factor 4E," Molecular and Cellular Biology, vol. 24, no. 1, pp. 200-216, 2004.

[18] J. H. Shin, S.-H. Min, S.-J. Kim et al., "TAK1 regulates autophagic cell death by suppressing the phosphorylation of p70 S6 kinase 1," Scientific Reports, vol. 3, article 1561, 2013.

[19] K.-S. Song, K. Jing, J.-S. Kim et al., “Omega-3-polyunsaturated fatty acids suppress pancreatic cancer cell growth in vitro and in vivo via downregulation of Wnt/beta-catenin signaling," Pancreatology, vol. 11, no. 6, pp. 574-584, 2012.

[20] S. E. Lee, J. W. Lim, and H. Kim, "Activator protein-1 mediates docosahexaenoic acid-induced apoptosis of human gastric cancer cells," Annals of the New York Academy of Sciences, vol. 1171, pp. 163-169, 2009.

[21] S. Serini, S. Trombino, F. Oliva et al., "Docosahexaenoic acid induces apoptosis in lung cancer cells by increasing MKP-1 and down-regulating p-ERK1/2 and p-p38 expression," Apoptosis, vol. 13, no. 9, pp. 1172-1183, 2008.

[22] J.-L. D. Toit-Kohn, L. Louw, and A.-M. Engelbrecht, "Docosahexaenoic acid induces apoptosis in colorectal carcinoma cells by modulating the PI3 kinase and p38 MAPK pathways," The Journal of Nutritional Biochemistry, vol. 20, no. 2, pp. 106-114, 2009.

[23] K. Jing, K.-S. Song, S. Shin et al., "Docosahexaenoic acid induces autophagy through p53/AMPK/mTOR signaling and promotes apoptosis in human cancer cells harboring wild-type p53," Autophagy, vol. 7, no. 11, pp. 1348-1358, 2011.

[24] S. Shin, K. Jing, S. Jeong et al., "The omega-3 polyunsaturated fatty acid DHA induces simultaneous apoptosis and autophagy via mitochondrial ROS-mediated Akt-mTOR signaling in prostate cancer cells expressing mutant p53," BioMed Research International, vol. 2013, Article ID 568671, 11 pages, 2013.

[25] K. D. Kikawa, J. S. Herrick, R. E. Tateo, M. Mouradian, J. S. Tay, and R. S. Pardini, "Induced oxidative stress and cell death in the A549 lung adenocarcinoma cell line by ionizing radiation is enhanced by supplementation with docosahexaenoic acid," Nutrition and Cancer, vol. 62, no. 8, pp. 1017-1024, 2010.
[26] C. Morin, S. Fortin, A. M. Cantin et al., "Anti-cancer effects of a new docosahexaenoic acid monoacylglyceride in lung adenocarcinoma," Recent Patents on Anti-Cancer Drug Discovery, vol. 8, no. 3, pp. 319-334, 2013.

[27] Q. H. Yao, X. C. Zhang, T. Fu et al., "Omega-3 polyunsaturated fatty acids inhibit the proliferation of the lung adenocarcinoma cell line A549 in vitro," Molecular Medicine Reports, vol. 9, pp. 401-406, 2014.

[28] E. Nam and C. Park, "Maspin suppresses survival of lung cancer cells through modulation of Akt pathway," Cancer Research and Treatment, vol. 42, no. 1, pp. 42-47, 2010.

[29] P. Cao, X. Cai, W. Lu, F. Zhou, and J. Huo, "Growth inhibition and induction of apoptosis in SHG-44 glioma cells by chinese medicine formula 'pingliu Keli"' Evidence-Based Complementary and Alternative Medicine, vol. 2011, Article ID 958243, 9 pages, 2011.

[30] S.-N. Sun, W.-D. Jia, H. Chen et al., "Docosahexaenoic acid (DHA) induces apoptosis in human hepatocellular carcinoma cells," International Journal of Clinical and Experimental Pathology, vol. 6, no. 2, pp. 281-289, 2013.

[31] S. Jeong, K. Jing, N. Kim et al., "Docosahexaenoic acid-induced apoptosis is mediated by activation of mitogen-activated protein kinases in human cancer cells," BMC Cancer, vol. 14, no. 1, article 481, 2014.

[32] H. Sheng, P. Li, X. Chen, B. Liu, Z. Zhu, and W. Cao, "Omega-3 PUFAs induce apoptosis of gastric cancer cells via ADORAl," Frontiers in Bioscience, vol. 19, pp. 854-861, 2014.

[33] G. Zhang, V. Gurtu, S. R. Kain, and G. Yan, "Early detection of apoptosis using a fluorescent conjugate of annexin V," BioTechniques, vol. 23, no. 3, pp. 525-531, 1997.

[34] Z. Darzynkiewicz, D. Galkowski, and H. Zhao, "Analysis of apoptosis by cytometry using TUNEL assay," Methods, vol. 44, no. 3, pp. 250-254, 2008.

[35] M. Su, Y. Mei, and S. Sinha, "Role of the crosstalk between autophagy and apoptosis in cancer," Journal of Oncology, vol. 2013, Article ID 102735, 14 pages, 2013.

[36] B. Cao, J. Li, X. Zhou et al., "Clioquinol induces pro-death autophagy in leukemia and myeloma cells by disrupting the mTOR signaling pathway," Scientific Reports, vol. 4, article 5749, 2014.

[37] J. J. Gills, J. Lopiccolo, and P. A. Dennis, "Nelfinavir, a new anticancer drug with pleiotropic effects and many paths to autophagy," Autophagy, vol. 4, no. 1, pp. 107-109, 2008.

[38] S. Wilkinson, J. O’Prey, M. Fricker, and K. M. Ryan, "Hypoxiaselective macroautophagy and cell survival signaled by autocrine PDGFR activity," Genes \& Development, vol. 23, no. 11, pp. 1283-1288, 2009.

[39] D.-D. Li, J.-F. Guo, J.-J. Huang et al., "Rhabdastrellic acid-A induced autophagy-associated cell death through blocking akt pathway in human cancer cells," PLoS ONE, vol. 5, no. 8, Article ID e12176, 2010.

[40] D. Gozuacik and A. Kimchi, "Autophagy and cell death," Current Topics in Developmental Biology, vol. 78, pp. 217-245, 2007.

[41] G. Xu, W. Zhang, P. Bertram, X. F. Zheng, and H. McLeod, "Pharmacogenomic profiling of the PI3K/PTEN-AKT-mTOR pathway in common human tumors," International Journal of Oncology, vol. 24, no. 4, pp. 893-900, 2004.

[42] A. M. Martelli, F. Chiarini, C. Evangelisti et al., "The phosphatidylinositol 3-kinase/AKT/mammalian target of rapamycin signaling network and the control of normal myelopoiesis," Histology and Histopathology, vol. 25, no. 5, pp. 669-680, 2010. 
[43] K. R. Rogers, K. D. Kikawa, M. Mouradian et al., "Docosahexaenoic acid alters epidermal growth factor receptor-related signaling by disrupting its lipid raft association," Carcinogenesis, vol. 31, no. 9, pp. 1523-1530, 2010.

[44] H. Xi, J. C. Barredo, J. R. Merchan, and T. J. Lampidis, "Endoplasmic reticulum stress induced by 2 -deoxyglucose but not glucose starvation activates AMPK through CaMKK $\beta$ leading to autophagy," Biochemical Pharmacology, vol. 85, no. 10, pp. 1463-1477, 2013.

[45] J. X. Kang, "Fat-1 transgenic mice: a new model for omega-3 research," Prostaglandins Leukotrienes \& Essential Fatty Acids, vol. 77, no. 5-6, pp. 263-267, 2007. 

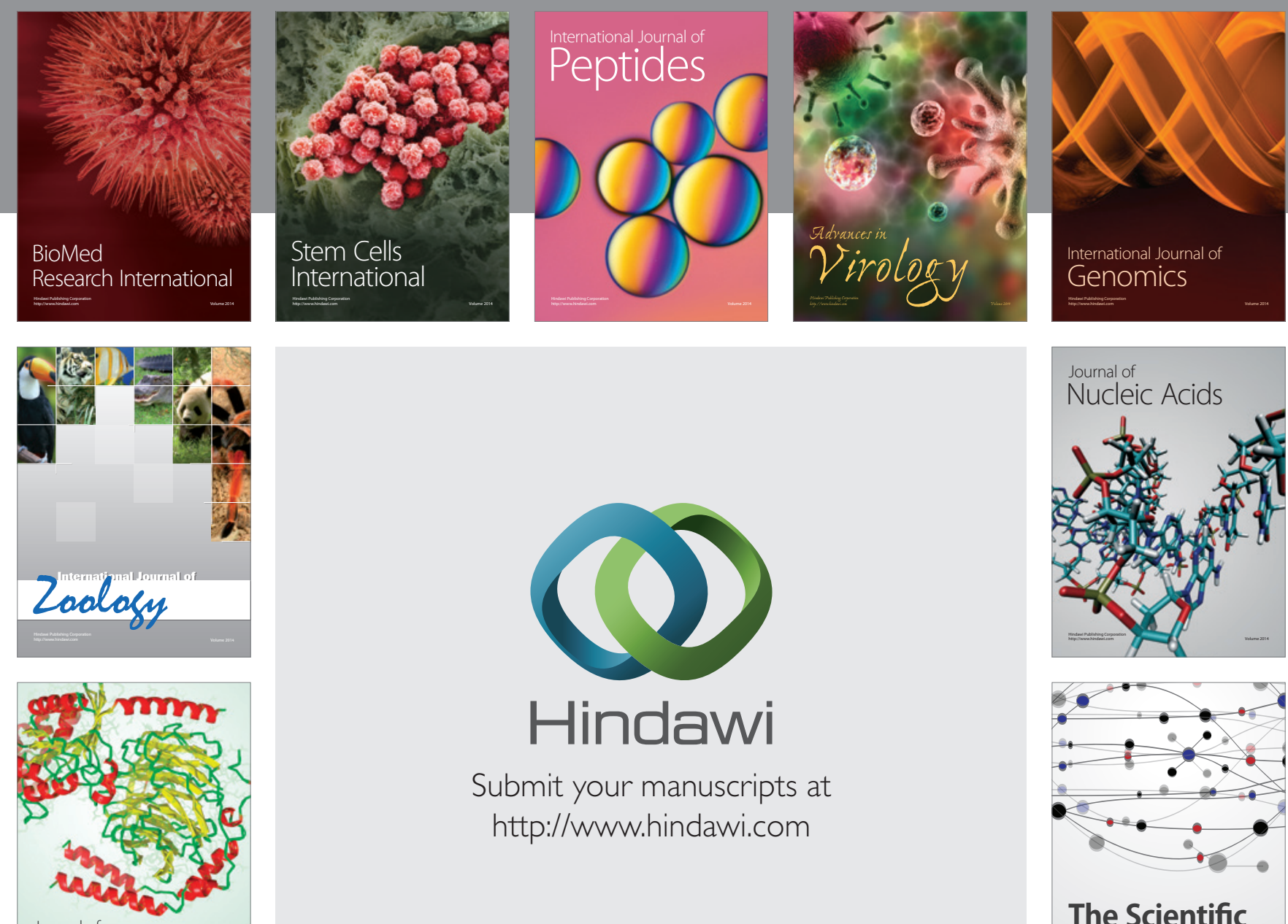

Submit your manuscripts at

http://www.hindawi.com

Journal of
Signal Transduction
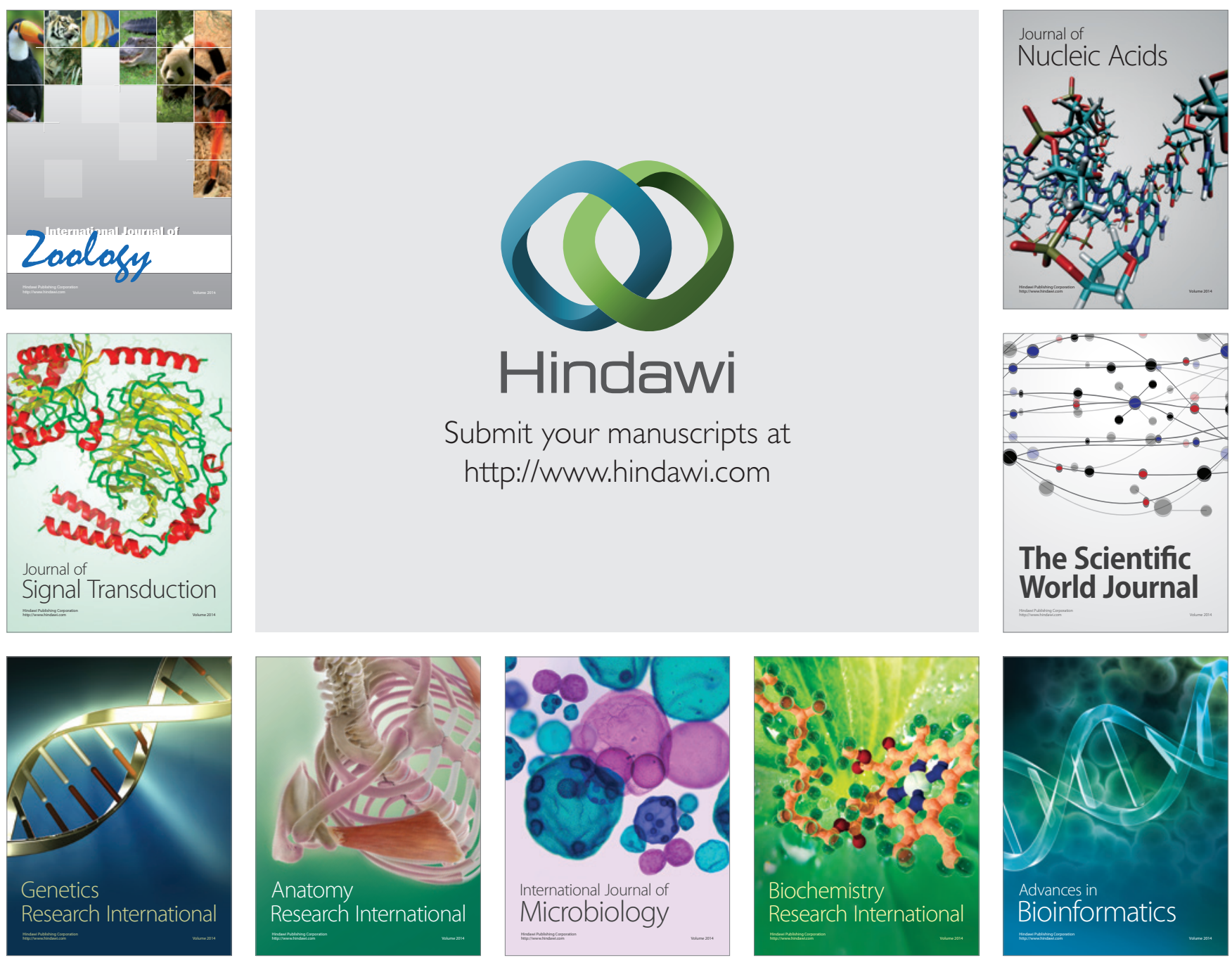

The Scientific World Journal
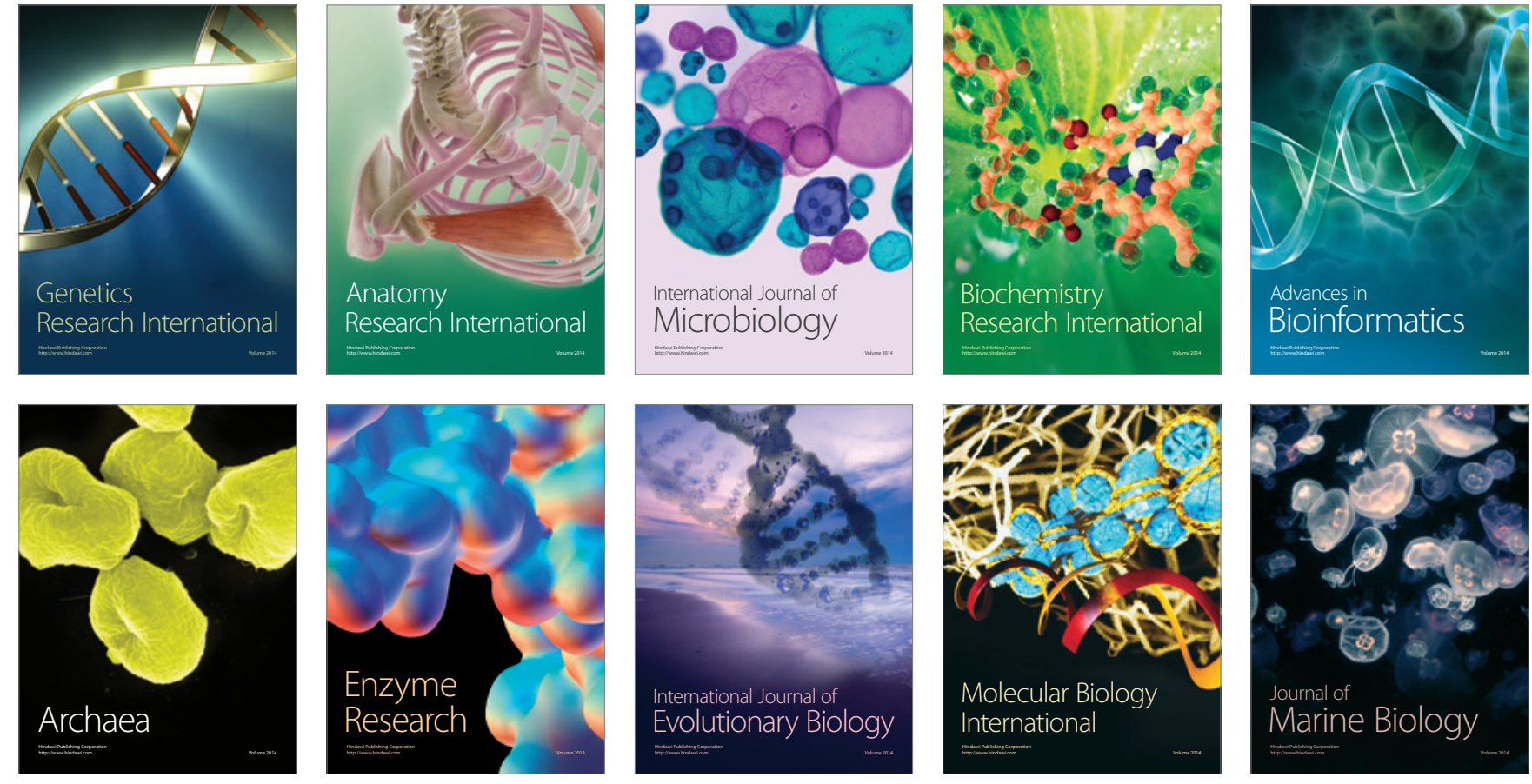